\title{
Stability analysis of delayed Takagi-Sugeno fuzzy systems: a new integral inequality approach
}

\author{
Jiyao $A n^{\mathrm{a}, *}$, Xinzhi Liub, Guilin Wen ${ }^{\mathrm{c}}$ \\ ${ }^{a}$ College of Computer Science and Electronic Engineering, Hunan University, Changsha 410082, China. \\ ${ }^{b}$ Department of Applied Mathematics, University of Waterloo, Waterloo, Ontario, Canada N2L 3G1. \\ ${ }^{c}$ State Key Laboratory of Advanced Design and Manufacture for Vehicle Body, College of Mechanical and Vehicle Engineering, Hunan \\ University, Changsha 410082, China.
}

Communicated by R. Saadati

\begin{abstract}
This paper is concerned with the problem of the stability analysis for Takagi-Sugeno (T-S) fuzzy systems with interval time-varying delay. The delay is assumed to be differential with interval bounds, and has both the lower and upper bounds of the delay derivatives, in which the upper bound of delay derivative may be greater than one. By constructing some delaydependent Lyapunov functions, some stability criteria are derived by using the convex optimization method and new integral inequality techniques. Utilizing integral inequalities for quadratic functions plays a key role in the field of stability analysis for delayed T-S fuzzy systems, and some integral inequalities for quadratic functions are derived and employed in order to produce tighter bounds than what the Jensen inequality and Wirtinger-based inequality produce. Then, less conservative stability criteria are derived by using convex combination method and improved integral inequalities based on appropriate Lyapunov-Krasovskii (LK) functional. Finally, several examples are given to show the advantages of the proposed results. (C) 2017 All rights reserved.
\end{abstract}

Keywords: T-S fuzzy systems, stability, interval time-varying delay, integral inequality, Lyapunov-Krasovskii (LK) functional. 2010 MSC: 37B25, 93C42, 93D20.

\section{Introduction}

Stability is a central issue in dynamical system and control theory. A dynamical system is called stable (in the sense of Lyapunov) if starting the system somewhere near its desired operating point implies that it will stay around that point ever after [8]. Nonlinear dynamics are almost ubiquitous in physical and engineering applications. The T-S fuzzy model [26] has been known to be a powerful tool because it can represent the system dynamics of nonlinear systems [6]. It is well-known that time delay is a source of instability or even oscillation and it often appears in engineering systems [6, 8], especially, the delay is assumed to be an interval time-varying delay $\tau(t)$, that is, $\tau(t) \in\left[h_{a}, h_{b}\right]$. Therefore, stability analysis for T-S fuzzy systems with interval time-varying delay is of great significance both in theory and in practice. As a result, the stability analysis and control synthesis for fuzzy systems with interval delay have been

\footnotetext{
*Corresponding author

Email addresses: anbobcn@aliyun.com (Jiyao An), xinzhi.liu@uwaterloo.ca (Xinzhi Liu), glwen@hnu.edu.cn (Guilin Wen)
} doi:10.22436/jnsa.010.04.53 
investigated in the past few decades, see $[2-4,7,9-13,15,17,18,21,22,24,25,27-29,31,32,34]$ and the references therein.

In the field of stability analysis, finding integral inequalities for quadratic functions plays a key role in the conservation of the stability conditions. There have been many approaches in the literature based on various mathematical tools such as (improved) Jensen inequality [1-4, 7, 15, 17, 18, 21, 24, 25, 29, 32, 32], free-weighting matrix method [13, 17, 29], reciprocally convex approach [16, 31-33] and fuzzy-weightingdependent Lyapunov function method [7, 28, 31, 34]. Recently, a delay partitioning approach has been proposed to further reduce their conservatism, see $[1,2,7,11,14,18,20,31,32,34]$ and references therein. Yang et al. [31] proposed an improved delay partitioning approach to stability analysis of delayed TS fuzzy systems. More recently, Zeng et al. [32] improved delay-dependent stability criteria for T-S fuzzy systems by a novel LK functional based on the idea of combining delay-decomposition with state vector augmentation and reciprocally convex approach, but the reciprocally convex method still has some conservatism according to the results in [33] and the decomposition approach is complicated [8].

Analyzing the conservatism of the Jensen inequality has been presented in [5] using the Gruss inequality, and an alternative inequality reducing the gap of the Jensen inequality has been proposed in [23] based on the Wirtinger inequality. Furthermore, compare to the Jensen inequality and Wirtinger inequality, a novel integral inequality so-called Bessel-Legendre (B-L) inequality has been developed in [24]. Very recently, some auxiliary function-based integral inequalities have been obtained and applied to stability analysis for time delay systems [17] which cover the Jensen inequality and the Wirtinger-based integral inequality. However, inequalities in $[23,24]$ only deal with single integral terms of quadratic functions while upper bounds of double integral terms can also be estimated if triple integral terms are introduced in the LK functional to reduce the conservatism [17]. Moreover, B-L inequality has only been applied to stability analysis of the system with constant delay [24] while the delay in [17] is assumed to be the fast time-varying delay. Utilizing the line-integral Lyapunov function and Wirtinger-based inequality in [34], less conservative conditions are obtained for time-delay T-S fuzzy systems by applying the fuzzy function approach [2] and the discretization technique [8], but the delay is assumed to be constant delay case. Therefore, there exists further room to investigate the upper bound of the time-derivative of the LK functional $[1,2,7,12,13,17,18,20-25,27,31-34]$, which motivates the present study.

We notice that delay-decomposition and (Jensen's) integral inequalities of stability analysis for systems with interval time-varying delays have not been investigated before as LMI-based cooperative optimization problems. Furthermore, all existing studies are on static delay partition which requires one delay to two equal subintervals or N-subintervals delay, or (improved) Jensen's inequality which has some constraint, that is, the Jensen gap in [5] is greater than a positive lower-bound [17, 23, 24, 34]. Our study in this paper makes some initial attempt to stability analysis for interval delayed T-S fuzzy systems. Although our modeling is for the T-S fuzzy systems with interval time-varying delays $[12,21,22,27]$, it is also applicable to the uncertain systems in terms of [1,18] and the networked control systems [3, 15, 19], while the proposed results can be extended to $\mathrm{H}_{\infty}$ control $[3,15,22,27]$.

Our contributions in this paper are summarized as follows:

(1) A new delay-dependent LK functional is constructed by developing a new delay-fractional-dependent partitioning approach.

(2) Based on Lemma 2.2, a tighter upper bound of the cross terms in the time-derivative of the LK functional is estimated by utilizing suitably convex combination technique, and then overall LMIbased sufficient conditions are derived without introducing any free-weighting matrices based on the Leibniz-Newton formula. Moreover, the proposed results depend on a delay decomposition tuning parameter $\delta$, and both the lower and upper bounds of delay and its derivative.

(3) Some triple terms in LK functionals are included, and the utilized state-augmented vector is extended to double integral terms, and then some integral inequalities in Lemma 2.3 give much tighter upper bounds than those obtained by Jensen inequality and Wirtinger-based inequality, especially for the estimation of double integral terms in the derivation process. Therefore, the resulting stability criterion 
in Theorem 3.1 is much less conservative than the ones based on Jensen inequality and Wirtinger-based inequality.

(4) Less conservatism than some existing ones is shown by some numerical examples, and the present results can be applied and effectuated to the case in which $d_{2} \geqslant 1$ (or fast time-varying delay in some existing ones).

Motivated by the above work, the aim of this paper is to study the problem of stability analysis for T-S fuzzy systems with interval time-varying delay by combining the delay-dependent LK functional approach with some new integral inequalities [17]. Firstly, we construct a novel delay-fractional-dependent LK functional included with some triple integral terms by developing a variable delay decomposition technique, in which the integral interval $\left[t-h_{b}, 0\right]$ is decomposed into $\left[t-h_{b}, t-\tau(t)\right],[t-\tau(t), t-\delta \tau(t)]$ and $\left[t-\delta \tau(t), t-\delta h_{a}\right],\left[t-\delta h_{a}, t\right]$ with $0<\delta<1$ as a tuning parameter. Secondly, compared to the Jensen inequality and Wirtinger-based inequality, the present inequalities have extra terms which can help to obtain much tighter bounds in the derivation process. Then, some less conservative delay-derivativedependent stability criteria are derived by employing convex optimization method in terms of linear matrix inequalities. The proposed results depend on a tuning parameter $\delta$, and both lower and upper bounds of time derivative of the delay. Finally, some numerical examples are used to compare with some previous results and demonstrate the merit of the proposed method.

\section{Problem formulation}

Consider a nonlinear system with interval time-varying delay via T-S fuzzy model, which can be described as follows:

Rule $j$ : If $\theta_{1}(t)$ is $N_{j 1}$ and $\ldots$ and $\theta_{p}(t)$ is $N_{j p}$, then

$$
\left\{\begin{array}{l}
\dot{x}(t)=A_{j} x(t)+A_{\tau j} x(t-\tau(t)), \\
x(t)=\phi(t), \forall t \in\left[-h_{b}, 0\right], j=1,2, \ldots, r,
\end{array}\right.
$$

where $0 \leqslant h_{a} \leqslant \tau(t) \leqslant h_{b}$, and $x(t)$ is the state vector; $\theta_{1}(t), \theta_{2}(t), \ldots, \theta_{p}(t)$ denote the premise variables while $N_{j 1}, N_{j 2}, \ldots, N_{j p}$ represent the fuzzy set. The system coefficient matrices are constant real matrices with appropriate dimensions, where $j=1,2 \ldots, r$ and $r$ is the number of IF-THEN rules. The initial function $\phi(t) \in W$, where $W$ is the space of absolutely continuous functions $\phi:\left[-h_{b}, 0\right] \rightarrow R^{n}$ with the square-integrable derivative and with appropriate norm.

The delay $\tau(t)$ is assumed to be time-varying delay as a differentiable function, satisfying

$$
0<h_{a} \leqslant \tau(t) \leqslant h_{b} \text {, and } d_{1} \leqslant \dot{\tau}(t) \leqslant d_{2},
$$

where $h_{a}, h_{b}$ and $d_{1}, d_{2}$ are some given values. For simplicity, we denote $\bar{\tau}=h_{b}-h_{a}, \tau_{0}=\frac{h_{a}^{2}}{2}, \tau_{s}=$ $\frac{\left(h_{b}-h_{a}\right)^{2}}{2}$.

The fuzzy system (2.1) is supposed to have singleton fuzzifier, product inference, and centroid defuzzifier. The final output of the fuzzy system is inferred as the following:

$$
\left\{\begin{array}{l}
\dot{x}(t)=A(t) x(t)+A_{\tau}(t) x(t-\tau(t)), \\
x(t)=\phi(t), \forall t \in\left[-h_{b}, 0\right]
\end{array}\right.
$$

where

$$
A(t):=\sum_{j=1}^{r} h_{j}(\theta(t)) A_{j}, A_{\tau}(t):=\sum_{j=1}^{r} h_{j}(\theta(t)) A_{\tau j}
$$

with $h_{j}(\theta(t))=\frac{\mu_{j}(\theta(t))}{\sum_{i=1}^{r} \mu_{i}(\theta(t))}, \mu_{j}(\theta(t))=\prod_{k=1}^{p} N_{j k}\left(\theta_{k}(t)\right), N_{j k}\left(\theta_{k}(t)\right)$ is the membership function of $\theta_{k}(t)$ in $N_{j k}$. Here $\mu_{j}(\theta(t)) \geqslant 0$, and $\sum_{j=1}^{r} h_{j}(\theta(t))=1$. 
The purpose of this paper is to find new stability criterion using new delay-decomposition and bounding techniques, which is less conservative than the existing results. Such a criterion may be used to compute the tolerable delay bound $h_{b}$ for given $h_{a}$ or vice versa.

To end this section, we introduce the following lemmas which are useful in stability analysis for the T-S fuzzy systems.

Lemma 2.1 (Moon et al. [14]). The following inequality holds for any $a \in \mathfrak{R}^{n_{a}}, b \in \mathfrak{R}^{n_{b}}, W \in \mathfrak{R}^{n_{a} \times n_{b}}$, $X \in \mathfrak{R}^{n_{\mathrm{a}} \times n_{\mathrm{b}}}, Y \in \mathfrak{R}^{n_{\mathrm{a}} \times \mathrm{n}_{\mathrm{b}}}$, and $\mathrm{Z} \in \mathfrak{R}^{\mathrm{n}_{\mathrm{b}} \times \mathrm{n}_{\mathrm{b}}}$

$$
-2 a^{\top} W b \leqslant\left[\begin{array}{l}
a \\
b
\end{array}\right]^{\top}\left[\begin{array}{cc}
X & Y-W \\
* & Z
\end{array}\right]\left[\begin{array}{l}
a \\
b
\end{array}\right]
$$

where $\left[\begin{array}{ll}\mathrm{X} & \mathrm{Y} \\ * & \mathrm{Z}\end{array}\right] \geqslant 0$.

Indentation on Lemma 2.1, we have the following integral inequality for quadratic integral terms.

Lemma 2.2. Let $x(t) \in \mathfrak{R}^{n}$ be a vector-valued function with first-order continuous-derivative entries. Then, for any matrices $M, N \in \mathfrak{R}^{n \times n}, Z \in \mathfrak{R}^{2 n \times 2 n}$, and some given scalars $0 \leqslant \tau_{1}<\tau_{2}$, and $\Lambda(s)=\Lambda(s)^{\top} \in \mathfrak{R}^{n \times n}$ is linear function in $\mathrm{s} \in\left[\mathrm{d}_{1}, \mathrm{~d}_{2}\right]$, the following integral inequalities hold:

1) When $\tau_{1}, \tau_{2}$ are time-varying, $h=\tau_{2}-\tau_{1}:=h(t) \geqslant 0$, and $\Lambda(s)$ is any symmetric matrix,

$$
-\int_{t-\tau_{2}}^{t-\tau_{1}} \dot{\chi}^{\top}(\theta) \Lambda(s) \dot{x}(\theta) d \theta \leqslant\left[\begin{array}{l}
x\left(t-\tau_{1}\right) \\
x\left(t-\tau_{2}\right)
\end{array}\right]^{\top}\left\{\left[\begin{array}{cc}
M+M^{\top} & -M+N^{\top} \\
* & -N-N^{\top}
\end{array}\right]+h Z\right\}\left[\begin{array}{l}
x\left(t-\tau_{1}\right) \\
x\left(t-\tau_{2}\right)
\end{array}\right]
$$

with $\left[\begin{array}{cc}\Lambda(s) & Y \\ * & \mathrm{Z}\end{array}\right] \geqslant 0$, and $\mathrm{Y}=\left[\begin{array}{ll}\mathrm{M} & \mathrm{N}\end{array}\right]$.

2) When $\tau_{1}, \tau_{2}$ are time-varying, $h=\tau_{2}-\tau_{1}:=h(t) \geqslant 0$, and $\Lambda(s)$ is a constant positive-definite matrix, that is $\Lambda(s)=\Lambda$,

$$
\begin{aligned}
-\int_{t-\tau_{2}}^{t-\tau_{1}} \dot{x}^{\top}(\theta) \Lambda \dot{x}(\theta) d \theta \leqslant & {\left[\begin{array}{l}
x\left(t-\tau_{1}\right) \\
x\left(t-\tau_{2}\right)
\end{array}\right]^{\top}\left\{\left[\begin{array}{cc}
M+M^{\top} & -M+N^{\top} \\
* & -N-N^{\top}
\end{array}\right]\right.} \\
& \left.+h\left[\begin{array}{c}
M \\
N
\end{array}\right] \Lambda^{-1}\left[M^{\top} \quad N^{\top}\right]\right\}\left[\begin{array}{l}
x\left(t-\tau_{1}\right) \\
x\left(t-\tau_{2}\right)
\end{array}\right] .
\end{aligned}
$$

Proof. From the Leibniz-Newton formula,

$$
0=x\left(t-\tau_{1}\right)-x\left(t-\tau_{2}\right)-\int_{t-\tau_{2}}^{t-\tau_{1}} \dot{x}(\theta) d \theta .
$$

So, the following equation holds for any $W_{1}, W_{2} \in \mathfrak{R}^{n \times n}$ :

$$
\begin{aligned}
0 & =2\left[x^{\top}\left(t-\tau_{1}\right) W_{1}^{\top}+x^{\top}\left(t-\tau_{2}\right) W_{2}^{\top}\right]\left[x\left(t-\tau_{1}\right)-x\left(t-\tau_{2}\right)-\int_{t-\tau_{2}}^{t-\tau_{1}} \dot{x}(\theta) d \theta\right] \\
& =2\left[\begin{array}{l}
x\left(t-\tau_{1}\right) \\
x\left(t-\tau_{2}\right)
\end{array}\right]^{\top}\left[\begin{array}{l}
W_{1}^{\top} \\
W_{2}^{\top}
\end{array}\right]\left[\begin{array}{ll}
I & -I
\end{array}\right]\left[\begin{array}{l}
x\left(t-\tau_{1}\right) \\
x\left(t-\tau_{2}\right)
\end{array}\right]-2 \int_{t-\tau_{2}}^{t-\tau_{1}}\left[\begin{array}{l}
x\left(t-\tau_{1}\right) \\
x\left(t-\tau_{2}\right)
\end{array}\right]^{\top}\left[\begin{array}{l}
W_{1}^{\top} \\
W_{2}^{\top}
\end{array}\right] \dot{x}(\theta) d \theta .
\end{aligned}
$$

Using Lemma 2.1 with $a:=\dot{x}(\theta)$ and $b:=\left[\begin{array}{l}x\left(t-\tau_{1}\right) \\ x\left(t-\tau_{2}\right)\end{array}\right]$ yields

$$
-2 \int_{t-\tau_{2}}^{t-\tau_{1}}\left[\begin{array}{l}
x\left(t-\tau_{1}\right) \\
x\left(t-\tau_{2}\right)
\end{array}\right]^{\top}\left[\begin{array}{l}
W_{1}^{\top} \\
W_{2}^{\top}
\end{array}\right] \dot{x}(\theta) d \theta \leqslant \int_{t-\tau_{2}}^{t-\tau_{1}} \dot{x}^{\top}(\theta) \Lambda(s) \dot{x}(\theta) d \theta
$$




$$
\begin{aligned}
& +2\left[\begin{array}{l}
x\left(t-\tau_{1}\right) \\
x\left(t-\tau_{2}\right)
\end{array}\right]^{\top}\left[\begin{array}{l}
M^{\top}-W_{1}^{\top} \\
N^{\top}-W_{2}^{\top}
\end{array}\right]\left[\begin{array}{ll}
I & -I
\end{array}\right]\left[\begin{array}{l}
x\left(t-\tau_{1}\right) \\
x\left(t-\tau_{2}\right)
\end{array}\right] \\
& +h\left[\begin{array}{l}
x\left(t-\tau_{1}\right) \\
x\left(t-\tau_{2}\right)
\end{array}\right]^{\top} Z\left[\begin{array}{l}
x\left(t-\tau_{1}\right) \\
x\left(t-\tau_{2}\right)
\end{array}\right] .
\end{aligned}
$$

So, combining with the above inequalities, we get the following inequality

$$
-\int_{t-\tau_{2}}^{t-\tau_{1}} \dot{x}^{\top}(\theta) \Lambda(s) \dot{x}(\theta) d \theta \leqslant 2\left[\begin{array}{l}
x\left(t-\tau_{1}\right) \\
x\left(t-\tau_{2}\right)
\end{array}\right]^{\top}\left[\begin{array}{l}
M^{\top} \\
N^{\top}
\end{array}\right]\left[\begin{array}{ll}
I & -I
\end{array}\right]\left[\begin{array}{l}
x\left(t-\tau_{1}\right) \\
x\left(t-\tau_{2}\right)
\end{array}\right]+h\left[\begin{array}{l}
x\left(t-\tau_{1}\right) \\
x\left(t-\tau_{2}\right)
\end{array}\right]^{\top} Z\left[\begin{array}{l}
x\left(t-\tau_{1}\right) \\
x\left(t-\tau_{2}\right)
\end{array}\right] .
$$

So far, a simple rearrangement for (2.5) yields (2.3). Furthermore, when $\Lambda(s)$ is a constant positive-definite matrix $\Lambda(s)=\Lambda$, we define $Z:=\left[\begin{array}{l}M^{\top} \\ N^{\top}\end{array}\right] \Lambda^{-1}\left[\begin{array}{ll}M & N\end{array}\right]$. Since that the following equation holds

$$
\left[\begin{array}{ll}
\Lambda & Y \\
* & Z
\end{array}\right]=\left[\begin{array}{cc}
\Lambda & Y \\
* & Y^{\top} \Lambda^{-1} Y
\end{array}\right]=G^{\top} G \geqslant 0
$$

where $G:=\left[\begin{array}{cc}\Lambda^{1 / 2} & \Lambda^{-1 / 2} Y \\ 0 & 0\end{array}\right]$, and $Y=\left[\begin{array}{ll}M & N\end{array}\right]$, the integral inequality (2.4) is true. This completes the proof.

Lemma 2.3 (Integral inequalities, Park [17]). Let $\chi(t) \in \mathfrak{R}^{n}$ be a vector-valued function with first-order continuous-derivative entries in $t \in[a, b]$. Then, $\mathrm{R}>0, \mathrm{R} \in \mathfrak{R}^{\mathrm{n} \times \mathrm{n}}$, the following single and double integral inequality holds:

$$
\begin{gathered}
-\int_{a}^{b} \dot{x}^{\top}(s) R \dot{x}(s) d s \leqslant-\frac{1}{b-a} \Delta_{1}^{\top} R \Delta_{1}-\frac{3}{b-a} \Delta_{2}^{\top} R \Delta_{2}-\frac{5}{b-a} \Delta_{3}^{\top} R \Delta_{3}, \\
-\int_{a}^{b} \int_{\theta}^{b} \dot{x}^{\top}(s) R \dot{x}(s) d s d \theta \leqslant-2 \Delta_{4}^{\top} R \Delta_{4}-4 \Delta_{5}^{\top} R \Delta_{5}, \\
-\int_{a}^{b} \int_{a}^{\theta} \dot{x}^{\top}(s) R \dot{x}(s) d s d \theta \leqslant-2 \Delta_{6}^{\top} R \Delta_{6}-4 \Delta_{7}^{\top} R \Delta_{7},
\end{gathered}
$$

where

$$
\begin{aligned}
& \Delta_{1}=x(b)-x(a) \\
& \Delta_{2}=x(b)+x(a)-\frac{2}{b-a} \int_{a}^{b} x(s) d s \\
& \Delta_{3}=x(b)-x(a)+\frac{6}{b-a} \int_{a}^{b} x(s) d s-\frac{12}{(b-a)^{2}} \int_{a}^{b} \int_{\theta}^{b} x(s) d s d \theta \\
& \Delta_{4}=x(b)-\frac{1}{b-a} \int_{a}^{b} x(s) d s, \\
& \Delta_{5}=x(b)+\frac{2}{b-a} \int_{a}^{b} x(s) d s-\frac{6}{(b-a)^{2}} \int_{a}^{b} \int_{\theta}^{b} x(s) d s d \theta \\
& \Delta_{6}=x(a)-\frac{1}{b-a} \int_{a}^{b} x(s) d s, \\
& \Delta_{7}=x(a)-\frac{4}{b-a} \int_{a}^{b} x(s) d s+\frac{6}{(b-a)^{2}} \int_{a}^{b} \int_{\theta}^{b} x(s) d s d \theta .
\end{aligned}
$$

\section{Main results}

This section aims to develop a novel delay decomposing approach for stability analysis of T-S fuzzy system (2.2). 
Firstly, we divide the delay interval $\left[0, h_{b}\right]$ into four segments: $\left[0, \delta h_{a}\right],\left[\delta h_{a}, \delta \tau(t)\right],[\delta \tau(t), \tau(t)]$, and $\left[\tau(t), h_{b}\right], 0<\delta<1$. For the sake of convenience, we denote

$$
\begin{aligned}
& \xi(t):=\operatorname{col}\left\{x(t) \quad x(t-\tau(t)) \quad x\left(t-\delta h_{a}\right) \quad x(t-\delta \tau(t)) \quad x\left(t-h_{a}\right) \quad x\left(t-h_{b}\right) \quad \dot{x}(t-\delta \tau(t)) \quad \xi_{0}(t)\right\}, \\
& \xi_{0}(t):=\operatorname{col}\left\{\frac{1}{h_{a}} \int_{t-h_{a}}^{t} x(s) d s \quad \frac{1}{\tau(t)-h_{a}} \int_{t-\tau(t)}^{t-h_{a}} x(s) d s \quad \frac{1}{h_{b}-\tau(t)} \int_{t-h_{b}}^{t-\tau(t)} x(s) d s\right. \\
& \left.\frac{2}{h_{a}^{2}} \int_{-h_{a}}^{0} \int_{t+\theta}^{t} x(s) d s d \theta \frac{2}{\left(\tau(t)-h_{a}\right)^{2}} \int_{-\tau(t)}^{-h_{a}} \int_{t+\theta}^{t-h_{a}} x(s) d s d \theta \frac{2}{\left(h_{b}-\tau(t)\right)^{2}} \int_{-h_{b}}^{-\tau(t)} \int_{t+\theta}^{t-\tau(t)} x(s) d s d \theta\right\}
\end{aligned}
$$

and $e_{i},(i=1,2, \ldots, 13)$ are block entry matrices, for example, $e_{1}=\left[\begin{array}{lllllllllllll}1 & 0 & 0 & 0 & 0 & 0 & 0 & 0 & 0 & 0 & 0 & 0 & 0\end{array}\right]$. For the T-S fuzzy system (2.2), based on the Lyapunov stability theorem, we will give some stability criteria by constructing a new LK functional and using a novel delay decomposition approach as follows:

Theorem 3.1. For given scalars $0<\mathrm{h}_{\mathrm{a}} \leqslant \mathrm{h}_{\mathrm{b}}, \mathrm{d}_{1} \leqslant \mathrm{~d}_{2}$, and $0<\delta<1$, satisfying $\delta \mathrm{d}_{2}<1$, the T-S fuzzy system (2.2) is asymptotically stable if there exist real symmetry matrices $\mathrm{P}^{\mathrm{i}}=\left[\begin{array}{cc}\mathrm{P}_{\mathrm{i} 1} & \mathrm{P}_{\mathrm{i} 2} \\ * & \mathrm{P}_{\mathrm{i} 3}\end{array}\right] \geqslant 0$ with $\mathrm{P}_{\mathrm{i} 1}>0,(i=1,2)$, $P_{\delta}>0, Q_{j}>0, R_{j}>0, S_{j}>0,(j=1,2,3,4), R_{s}>0$, and any matrices $\Theta=\left[\Theta_{i j}\right]_{3 \times 3}, Z_{j}=\left[\begin{array}{cc}Z_{j 1} & Z_{j 2} \\ * & Z_{j 3}\end{array}\right]$, $(j=1,2,3), M_{i}, N_{i},(i=1,2,3,4)$ with appropriate dimensions such that the LMIs in (3.1)-(3.4) when $\tau(t) \rightarrow h_{a}$ and $\tau(\mathrm{t}) \rightarrow \mathrm{h}_{\mathrm{b}}$ hold, for any $\mathrm{l}=1,2, \ldots, \mathrm{r}$.

$$
\Xi_{l}\left(\tau(t), d_{1}\right)<0 \text { and } \Xi_{l}\left(\tau(t), d_{2}\right)<0
$$

with

$$
\begin{aligned}
& {\left[\begin{array}{cc}
d_{k} R_{4}+\left(1-d_{k}\right) R_{3} & {\left[\begin{array}{cc}
M_{2} & N_{2}
\end{array}\right]} \\
* & Z_{3}
\end{array}\right] \geqslant 0,} \\
& {\left[\begin{array}{cc}
d_{k} R_{4}+(\delta-1) d_{k} R_{3}+\left(1-\delta d_{k}\right) R_{2} & {\left[\begin{array}{cc}
M_{3} & N_{3}
\end{array}\right]} \\
* & Z_{2}
\end{array}\right] \geqslant 0,} \\
& {\left[\begin{array}{cc}
d_{k} R_{4}+(\delta-1) d_{k} R_{3}-\delta d_{k} R_{2}+R_{1} & {\left[\begin{array}{cc}
M_{4} & N_{4} \\
Z_{1}
\end{array}\right] \geqslant 0, k=1,2,}
\end{array}\right.}
\end{aligned}
$$

and

$$
\left[\begin{array}{cc}
\mathrm{RS}_{3} & \Theta \\
* & \mathrm{RS}_{4}
\end{array}\right]>0
$$

where

$$
\begin{aligned}
& \Xi_{l}(\tau(t), \dot{\tau}(t))=\zeta_{0}^{\top} \Xi_{0} \zeta_{0}+\zeta_{1}^{\top} \mathrm{P}(\tau(\mathrm{t})) \zeta_{2}+\zeta_{2}^{\mathrm{T}} \mathrm{P}(\tau(\mathrm{t})) \zeta_{1}+(1-\delta \dot{\tau}(\mathrm{t}))\left[\zeta_{1}^{\mathrm{T}} \mathrm{P}(\tau(\mathrm{t})) \zeta_{3}+\zeta_{3}^{\mathrm{T}} \mathrm{P}(\tau(\mathrm{t})) \zeta_{1}-e_{7}^{\mathrm{T}} \mathrm{P}_{\delta} e_{7}\right] \\
& +\zeta_{1}^{\mathrm{T}}\left[\frac{\dot{\tau}(\mathrm{t})}{\bar{\tau}}\left(\mathrm{P}^{1}-\mathrm{P}^{2}\right)\right] \zeta_{1}-\left\{2\left(e_{1}-e_{8}\right)^{\mathrm{T}} S_{1}\left(e_{1}-e_{8}\right)+4\left(e_{1}+2 e_{8}-3 e_{11}\right)^{\mathrm{T}} S_{1}\left(e_{1}+2 e_{8}-3 e_{11}\right)\right. \\
& +2\left(e_{8}-e_{5}\right)^{\mathrm{T}} S_{2}\left(e_{8}-e_{5}\right)+4\left(e_{5}-4 e_{8}+3 e_{11}\right)^{\mathrm{T}} S_{2}\left(e_{5}-4 e_{8}+3 e_{11}\right) \\
& +2\left(e_{5}-e_{9}\right)^{\top} S_{3}\left(e_{5}-e_{9}\right)+4\left(e_{5}+2 e_{9}-3 e_{12}\right)^{\top} S_{3}\left(e_{5}+2 e_{9}-3 e_{12}\right) \\
& +2\left(e_{2}-e_{10}\right)^{\top} S_{3}\left(e_{2}-e_{10}\right)+4\left(e_{2}+2 e_{10}-3 e_{13}\right)^{\top} S_{3}\left(e_{2}+2 e_{10}-3 e_{13}\right) \\
& +2\left(e_{9}-e_{2}\right)^{\top} S_{4}\left(e_{9}-e_{2}\right)+4\left(e_{2}-4 e_{9}+3 e_{12}\right)^{\top} S_{4}\left(e_{2}-4 e_{9}+3 e_{12}\right) \\
& \left.+2\left(e_{10}-e_{6}\right)^{\top} S_{4}\left(e_{10}-e_{6}\right)+4\left(e_{6}-4 e_{10}+3 e_{13}\right)^{\top} S_{4}\left(e_{6}-4 e_{10}+3 e_{13}\right)\right\} \\
& +e_{1}^{\top} Q_{1} e_{1}+e_{3}^{\top}\left(Q_{2}-Q_{1}\right) e_{3}+(1-\delta \dot{\tau}(t)) e_{4}^{T}\left(Q_{3}-Q_{2}\right) e_{4}+(1-\dot{\tau}(t)) e_{2}^{\top}\left(Q_{4}-Q_{3}\right) e_{2}-e_{5}^{T} Q_{4} e_{5} \\
& +\left[\begin{array}{l}
e_{2} \\
e_{6}
\end{array}\right]^{\top}\left\{M N_{1}+\left(h_{b}-\tau(t)\right)\left[\begin{array}{c}
M_{1} \\
N_{1}
\end{array}\right] R_{4}^{-1}\left[\begin{array}{ll}
M_{1}^{\top} & N_{1}^{\top}
\end{array}\right]\right\}\left[\begin{array}{l}
e_{2} \\
e_{6}
\end{array}\right] \\
& +\left[\begin{array}{l}
e_{4} \\
e_{2}
\end{array}\right]^{\top}\left\{M N_{2}+(1-\delta) \tau(t) Z_{3}\right\}\left[\begin{array}{l}
e_{4} \\
e_{2}
\end{array}\right]
\end{aligned}
$$




$$
\begin{aligned}
& +\left[\begin{array}{l}
e_{3} \\
e_{4}
\end{array}\right]^{\top}\left\{M N_{3}+\delta\left(\tau(t)-h_{a}\right) Z_{2}\right\}\left[\begin{array}{l}
e_{3} \\
e_{4}
\end{array}\right]+\left[\begin{array}{l}
e_{1} \\
e_{3}
\end{array}\right]^{\top}\left\{M N_{4}+\delta h_{a} Z_{1}\right\}\left[\begin{array}{l}
e_{1} \\
e_{3}
\end{array}\right]-\Psi, \\
& \Psi=\Gamma\left[\begin{array}{cc}
\operatorname{diag}\left\{R_{s}, 3 R_{s}, 5 R_{s}\right\} & \Theta \\
* & \operatorname{diag}\left\{R_{s}, 3 R_{s}, 5 R_{s}\right\}
\end{array}\right] \Gamma^{\top}, \\
& \Gamma=\left[\begin{array}{lll}
\left(e_{5}-e_{2}\right. & )^{\top} & \left(e_{5}+e_{2}-2 e_{9}\right.
\end{array}\right)^{\top} \quad\left(e_{5}-e_{2}+6 e_{9}-6 e_{12}\right)^{\top} \\
& \left.\times\left(e_{2}-e_{6}\right)^{\top} \quad\left(e_{2}+e_{6}-2 e_{10}\right)^{\top} \quad\left(e_{2}-e_{6}+6 e_{10}-6 e_{13}\right)^{\top}\right], \\
& M N_{k}=\left[\begin{array}{cc}
M_{k}+M_{k}^{\top} & -M_{k}+N_{k}^{\top} \\
* & -N_{k}-N_{k}^{\top}
\end{array}\right],(k=1,2,3,4), \\
& P(\tau(t))=\frac{\tau(t)-h_{a}}{\tau} P^{1}+\frac{h_{b}-\tau(t)}{\tau} P^{2}, \\
& R_{3}=\operatorname{diag}\left\{R_{s}+S_{3} \quad 3\left(R_{s}+S_{3}\right) \quad 5\left(R_{s}+S_{3}\right)\right\}, \\
& \mathrm{RS}_{4}=\operatorname{diag}\left\{\mathrm{R}_{\mathrm{s}}+\mathrm{S}_{4} \quad 3\left(\mathrm{R}_{\mathrm{s}}+\mathrm{S}_{4}\right) \quad 5\left(\mathrm{R}_{\mathrm{s}}+\mathrm{S}_{4}\right)\right\} \text {, } \\
& \Xi_{0}:=P_{\delta}+\delta h_{a} R_{1}+\delta\left(\tau(t)-h_{a}\right) R_{2}+(1-\delta) \tau(t) R_{3}+\left(h_{b}-\tau(t)\right) R_{4}+\tau_{0} S_{1} \\
& +\tau_{0} S_{2}+\tau_{s} S_{3}+\tau_{s} S_{4}+\bar{\tau}^{2} R_{s} \text {, } \\
& \xi_{0}=A_{l} e_{1}+A_{\tau l} e_{2}, \xi_{1}=\left(\begin{array}{c}
e_{1} \\
e_{4}
\end{array}\right), \xi_{2}=\left(\begin{array}{c}
\xi_{0} \\
0
\end{array}\right), \xi_{3}=\left(\begin{array}{c}
0 \\
e_{7}
\end{array}\right) \text {. }
\end{aligned}
$$

Proof. Choose the following delay-dependent LK functional:

$$
V\left(t, x_{t}, \dot{x}_{t}\right)=V_{1}\left(t, x_{t}, \dot{x}_{t}\right)+V_{2}\left(t, x_{t}, \dot{x}_{t}\right)+V_{3}\left(t, x_{t}, \dot{x}_{t}\right)+V_{4}\left(t, x_{t}, \dot{x}_{t}\right),
$$

where $x_{t}$ denotes the function $x(s)$ defined on the interval $\left[t-h_{b}, t\right]$, and

$$
\begin{aligned}
& V_{1}\left(t, x_{t}, \dot{x}_{t}\right)=\left[\begin{array}{c}
x(t) \\
x(t-\delta \tau(t))
\end{array}\right]^{\top} P(\tau(t))\left[\begin{array}{c}
x(t) \\
x(t-\delta \tau(t))
\end{array}\right]+\int_{t-\delta \tau(t)}^{t} \dot{x}^{\top}(s) P_{\delta} \dot{x}(s) d s, \\
& P(\tau(t))=\frac{\tau(t)-h_{a}}{\tau} P^{1}+\frac{h_{b}-\tau(t)}{\tau} P^{2}, \\
& V_{2}\left(t, x_{t}, \dot{x}_{t}\right)=\int_{t-\delta h_{a}}^{t} x^{\top}(s) Q_{1} x(s) d s+\int_{t-\delta \tau(t)}^{t-\delta h_{a}} x^{\top}(s) Q_{2} x(s) d s \\
& +\int_{t-\tau(t)}^{t-\delta \tau(t)} \chi^{\top}(s) Q_{3} x(s) d s+\int_{t-h_{b}}^{t-\tau(t)} \chi^{\top}(s) Q_{4} x(s) d s, \\
& V_{3}\left(t, x_{t}, \dot{x}_{t}\right)=\int_{-\delta h_{a}}^{0} \int_{t+\theta}^{t} \dot{x}^{\top}(s) R_{1} \dot{x}(s) d s d \theta+\int_{-\delta \tau(t)}^{-\delta h_{a}} \int_{t+\theta}^{t} \dot{x}^{\top}(s) R_{2} \dot{x}(s) d s d \theta \\
& +\int_{-\tau(t)}^{-\delta \tau(t)} \int_{t+\theta}^{t} \dot{x}^{\top}(s) R_{3} \dot{x}(s) d s d \theta+\int_{-h_{b}}^{-\tau(t)} \int_{t+\theta}^{t} \dot{x}^{\top}(s) R_{4} \dot{x}(s) d s d \theta \\
& V_{4}\left(t, x_{t}, \dot{x}_{t}\right)=\int_{-h_{a}}^{0} \int_{\theta}^{0} \int_{t+\lambda}^{t} \dot{x}^{\top}(s) S_{1} \dot{x}(s) d s d \lambda d \theta+\int_{-h_{a}}^{0} \int_{-h_{a}}^{\theta} \int_{t+\lambda}^{t} \dot{x}^{\top}(s) S_{2} \dot{x}(s) d s d \lambda d \theta \\
& +\int_{-h_{b}}^{-h_{a}} \int_{\theta}^{-h_{a}} \int_{t+\lambda}^{t} \dot{x}^{\top}(s) S_{3} \dot{x}(s) d s d \lambda d \theta+\int_{-h_{b}}^{-h_{a}} \int_{-h_{b}}^{\theta} \int_{t+\lambda}^{t} \dot{x}^{\top}(s) S_{4} \dot{x}(s) d s d \lambda d \theta \\
& +\bar{\tau} \int_{-h_{b}}^{-h_{a}} \int_{t+\theta}^{t} \dot{x}^{\top}(s) R_{5} \dot{x}(s) d s d \theta,
\end{aligned}
$$

with $\left[\begin{array}{cc}P_{i 1} & P_{i 2} \\ * & P_{i 3}\end{array}\right] \geqslant 0$ with $P_{i 1}>0,(i=1,2), P_{\delta}>0, Q_{j}>0, R_{j}>0, S_{j}>0,(j=1,2,3,4), R_{s}>0$ being real symmetry matrices. 
Note that the function $P(\tau(t))$ is continuous in $t$, and $\lim _{\tau(t) \rightarrow h_{a}} P(\tau(t))=P^{2}$ and $\lim _{\tau(t) \rightarrow h_{b}} P(\tau(t))=$ $\mathrm{P}^{1}$. In the following [9], we define the derivative form as

$$
\dot{V}\left(t, x_{t}, \dot{x}_{t}\right)=\lim _{s \rightarrow 0^{+}} \frac{1}{s}\left[V\left(t+s, x_{t+s}, \dot{x}_{t+s}\right)-V\left(t, x_{t}, \dot{x}_{t}\right)\right]
$$

And then we are seeking for conditions guaranteeing that $\dot{V}\left(t, x_{t}\right) \leqslant-\varepsilon\|x(t)\|^{2}$ for a sufficiently small $\varepsilon>0$.

$$
\begin{aligned}
& \dot{V}_{1}\left(t, x_{t}, \dot{x}_{t}\right)=\left[\begin{array}{c}
\dot{x}(t) \\
\dot{x}(t-\delta \tau(t))
\end{array}\right]^{\top} P(\tau(t))\left[\begin{array}{c}
x(t) \\
x(t-\delta \tau(t))
\end{array}\right]+\left[\begin{array}{c}
x(t) \\
x(t-\delta \tau(t))
\end{array}\right]^{\top} P(\tau(t))\left[\begin{array}{c}
\dot{x}(t) \\
\dot{x}(t-\delta \tau(t))
\end{array}\right] \\
& +\left[\begin{array}{c}
x(t) \\
x(t-\delta \tau(t))
\end{array}\right]^{\top} \dot{P}(\tau(t))\left[\begin{array}{c}
x(t) \\
x(t-\delta \tau(t))
\end{array}\right] \\
& +\dot{x}^{\top}(t) P_{\delta} \dot{x}(t)-(1-\delta \tau(t)) \dot{x}^{\top}(t-\tau(t)) P_{\delta} \dot{x}(t-\tau(t)) \\
& =\xi^{\top}(\mathrm{t})\left\{\zeta_{0}^{\top} \mathrm{P}_{\delta} \zeta_{0}+\zeta_{1}^{\top} \mathrm{P}(\tau(\mathrm{t})) \zeta_{2}+\zeta_{2}^{\mathrm{T}} \mathrm{P}(\tau(\mathrm{t})) \zeta_{1}\right. \\
& +(1-\delta \dot{\tau}(t))\left[\zeta_{1}^{\top} \mathrm{P}(\tau(\mathrm{t})) \zeta_{3}+\zeta_{3}^{\mathrm{T}} \mathrm{P}(\tau(\mathrm{t})) \zeta_{1}-e_{6}^{\mathrm{T}} \mathrm{P}_{\delta} \mathrm{e}_{6}\right] \\
& \left.+\zeta_{1}^{\top}\left[\frac{\dot{\tau}(\mathrm{t})}{\bar{\tau}\left(\mathrm{P}^{1}-\mathrm{P}^{2}\right)}\right] \zeta_{1}\right\} \xi(\mathrm{t}), \zeta_{0}=A(\mathrm{t}) e_{1}+A_{\tau}(\mathrm{t}) e_{2}, \\
& \dot{V}_{2}\left(t, x_{t}, \dot{x}_{t}\right)=\xi^{\top}(t)\left\{e_{1}^{\top} Q_{1} e_{1}+e_{3}^{\top}\left(Q_{2}-Q_{1}\right) e_{3}+(1-\delta \dot{\tau}(t)) e_{4}^{\top}\left(Q_{3}-Q_{2}\right) e_{4}\right. \\
& \left.+(1-\dot{\tau}(\mathrm{t})) e_{2}^{\top}\left(\mathrm{Q}_{4}-\mathrm{Q}_{3}\right) e_{2}-e_{5}^{\mathrm{T}} \mathrm{Q}_{4} e_{5}\right\} \xi(\mathrm{t}), \\
& \dot{V}_{3}\left(t, x_{t}, \dot{x}_{t}\right)=\xi(t) \zeta_{0}^{\top} \Xi_{00} \zeta_{0} \xi(t)-\int_{t-h_{b}}^{t-\tau(t)} \dot{x}^{\top}(s) R_{4} \dot{x}(s) d s \\
& -\int_{t-\tau(t)}^{t-\delta \tau(t)} \dot{x}^{\top}(s)\left[\dot{\tau}(t) R_{4}+(1-\dot{\tau}(t)) R_{3}\right] \dot{x}(s) d s \\
& -\int_{t-\delta \tau(t)}^{t-\delta h_{a}} \dot{x}^{\top}(s)\left[\dot{\tau}(t) R_{4}+(\delta-1) \dot{\tau}(t) R_{3}+(1-\delta \dot{\tau}(t)) R_{2}\right] \dot{x}(s) d s \\
& -\int_{t-\delta h_{a}}^{t} \dot{x}^{\top}(s)\left[\dot{\tau}(t) R_{4}+(\delta-1) \dot{\tau}(t) R_{3}-\delta \dot{\tau}(t) R_{2}+R_{1}\right] \dot{x}(s) d s, \\
& \dot{V}_{4}\left(t, x_{t}, \dot{x}_{t}\right)=\dot{\chi}^{\top}(t)\left[\tau_{0} S_{1}+\tau_{0} S_{2}\right] \dot{x}(t)-\int_{-h_{a}}^{0} \int_{t+\theta}^{t} \dot{x}^{\top}(s) S_{1} \dot{x}(s) d s d \theta \\
& -\int_{-h_{a}}^{0} \int_{t-h_{a}}^{t+\theta} \dot{x}^{\top}(s) S_{2} \dot{x}(s) d s d \theta+\dot{x}^{\top}(t)\left[\tau_{s} S_{3}+\tau_{s} S_{4}\right] \dot{x}(t) \\
& -\int_{-h_{b}}^{-h_{a}} \int_{t+\theta}^{t-h_{a}} \dot{\chi}^{\top}(s) S_{3} \dot{x}(s) d s d \theta-\int_{-h_{b}}^{-h_{a}} \int_{t-h_{b}}^{t+\theta} \dot{\chi}^{\top}(s) S_{4} \dot{x}(s) d s d \theta \\
& +\dot{x}^{\top}(t)\left[\bar{\tau}^{2} R_{s}\right] \dot{x}(t)-\bar{\tau} \int_{t-\tau(t)}^{t-h_{a}} \dot{x}^{\top}(s) R_{s} \dot{x}(s) d s d \theta-\bar{\tau} \int_{t-h_{b}}^{t-\tau(t)} \dot{x}^{\top}(s) R_{s} \dot{x}(s) d s d \theta \\
& =\zeta_{0}^{\top}\left[\tau_{0} S_{1}+\tau_{0} S_{2}+\tau_{s} S_{3}+\tau_{s} S_{4}+\bar{\tau}^{2} R_{s}\right] \zeta_{0}-\int_{-h_{a}}^{0} \int_{t+\theta}^{t} \dot{x}^{\top}(s) S_{1} \dot{x}(s) d s d \theta \\
& -\int_{-h_{a}}^{0} \int_{t-h_{a}}^{t+\theta} \dot{x}^{\top}(s) S_{2} \dot{x}(s) d s d \theta-\int_{-\tau(t)}^{-h_{a}} \int_{t+\theta}^{t-h_{a}} \dot{x}^{\top}(s) S_{3} \dot{x}(s) d s d \theta
\end{aligned}
$$




$$
\begin{aligned}
& -\int_{-h_{b}}^{-\tau(t)} \int_{t+\theta}^{t-\tau(t)} \dot{x}^{\top}(s) S_{3} \dot{x}(s) d s d \theta-\left(h_{b}-\tau(t)\right) \int_{t-\tau(t)}^{t-h_{a}} \dot{x}^{\top}(s) S_{3} \dot{x}(s) d s \\
& -\int_{-\tau(t)}^{-h_{a}} \int_{t-\tau(t)}^{t+\theta} \dot{x}^{\top}(s) S_{4} \dot{x}(s) d s d \theta-\int_{-h_{b}}^{-\tau(t)} \int_{t-h_{b}}^{t+\theta} \dot{x}^{\top}(s) S_{4} \dot{x}(s) d s d \theta \\
& -\left(\tau(t)-h_{a}\right) \int_{t-h_{b}}^{t-\tau(t)} \dot{x}^{\top}(s) S_{4} \dot{x}(s) d s-\bar{\tau} \int_{t-\tau(t)}^{t-h_{a}} \dot{x}^{\top}(s) R_{s} \dot{x}(s) d s d \theta \\
& -\bar{\tau} \int_{t-h_{b}}^{t-\tau(t)} \dot{x}^{\top}(s) R_{s} \dot{x}(s) d s d \theta,
\end{aligned}
$$

where $\zeta_{1}, \zeta_{2}, \zeta_{3}$ are defined in (3.5) and $\Xi_{00}=\delta h_{a} R_{1}+\delta\left(\tau(t)-h_{a}\right) R_{2}+(1-\delta) \tau(t) R_{3}+\left(h_{b}-\tau(t)\right) R_{4}$.

Using Lemma 2.2, one can get the following inequalities

$$
\begin{aligned}
& -\int_{t-h_{b}}^{t-\tau(t)} \dot{x}^{\top}(s) R_{4} \dot{x}(s) d s \leqslant\left[\begin{array}{c}
x(t-\tau(t)) \\
x\left(t-h_{b}\right)
\end{array}\right]^{\top}\left\{\left[\begin{array}{cc}
M_{1}+M_{1}^{\top} & -M_{1}+N_{1}^{\top} \\
* & -N_{1}-N_{1}^{\top}
\end{array}\right]\right. \\
& \left.+\left(h_{b}-\tau(t)\right) \cdot\left[\begin{array}{c}
M_{1} \\
N_{1}
\end{array}\right] R_{4}^{-1}\left[\begin{array}{c}
M_{1} \\
N_{1}
\end{array}\right]^{\top}\right\}\left[\begin{array}{c}
x(t-\tau(t)) \\
x\left(t-h_{b}\right)
\end{array}\right] \\
& =\xi^{\top}(t)\left\{[ \begin{array} { l } 
{ e _ { 2 } } \\
{ e _ { 5 } }
\end{array} ] ^ { \top } \left[\left[\begin{array}{cc}
M_{1}+M_{1}^{\top} & -M_{1}+N_{1}^{\top} \\
* & -N_{1}-N_{1}^{\top}
\end{array}\right]\right.\right. \\
& \left.\left.+\left(h_{b}-\tau(t)\right) \cdot\left[\begin{array}{l}
M_{1} \\
N_{1}
\end{array}\right] R_{4}^{-1}\left[\begin{array}{l}
M_{1} \\
N_{1}
\end{array}\right]^{\top}\right]\left[\begin{array}{l}
e_{2} \\
e_{5}
\end{array}\right]\right\} \xi(t), \\
& -\int_{t-\tau(t)}^{t-\delta \tau(t)} \dot{x}^{\top}(s)\left[\dot{\tau}(t) R_{4}+(1-\dot{\tau}(t)) R_{3}\right] \dot{x}(s) d s \\
& \leqslant\left[\begin{array}{c}
x(t-\delta \tau(t)) \\
x(t-\tau(t))
\end{array}\right]^{\top}\left\{\left[\begin{array}{cc}
M_{2}+M_{2}^{\top} & -M_{2}+N_{2}^{\top} \\
* & -N_{2}-N_{2}^{\top}
\end{array}\right]+(1-\delta) \tau(t) Z_{3}\right\}\left[\begin{array}{c}
x(t-\delta \tau(t)) \\
x(t-\tau(t))
\end{array}\right] \\
& =\xi^{\top}(t)\left\{\left[\begin{array}{l}
e_{4} \\
e_{2}
\end{array}\right]^{\top}\left[\left[\begin{array}{cc}
M_{2}+M_{2}^{\top} & -M_{2}+N_{2}^{\top} \\
* & -N_{2}-N_{2}^{\top}
\end{array}\right]+(1-\delta) \tau(t) Z_{3}\right]\left[\begin{array}{l}
e_{4} \\
e_{2}
\end{array}\right]\right\} \xi(t),
\end{aligned}
$$

with $\left[\begin{array}{cc}\dot{\tau}(t) R_{4}+(1-\dot{\tau}(t)) R_{3} & {\left[\begin{array}{cc}M_{2} & N_{2} \\ Z_{3}\end{array}\right] \geqslant 0 .}\end{array}\right.$

Similarly, we have

$$
\begin{aligned}
& -\int_{t-\delta \tau(t)}^{t-\delta h_{a}} \dot{x}^{\top}(s)\left[\dot{\tau}(t) R_{4}+(\delta-1) \dot{\tau}(t) R_{3}+(1-\delta \dot{\tau}(t)) R_{2}\right] \dot{x}(s) d s \\
& \quad \leqslant\left[\begin{array}{c}
x\left(t-\delta h_{a}\right) \\
x(t-\delta \tau(t))
\end{array}\right]^{\top}\left\{\left[\begin{array}{cc}
M_{3}+M_{3}^{\top} & -M_{3}+N_{3}^{\top} \\
* & -N_{3}-N_{3}^{T}
\end{array}\right]+\delta\left(\tau(t)-h_{a}\right) Z_{2}\right\}\left[\begin{array}{c}
x\left(t-\delta h_{a}\right) \\
x(t-\delta \tau(t))
\end{array}\right] \\
& \quad=\xi^{\top}(t)\left\{\left[\begin{array}{c}
e_{3} \\
e_{4}
\end{array}\right]^{\top}\left[\left[\begin{array}{cc}
M_{3}+M_{3}^{\top} & -M_{3}+N_{3}^{\top} \\
* & -N_{3}-N_{3}^{T}
\end{array}\right]+\delta\left(\tau(t)-h_{a}\right) Z_{2}\right]\left[\begin{array}{c}
e_{3} \\
e_{4}
\end{array}\right]\right\} \xi(t)
\end{aligned}
$$

and

$$
\begin{aligned}
& -\int_{t-\delta h_{a}}^{t} \dot{x}^{\top}(s)\left[\dot{\tau}(t) R_{4}+(\delta-1) \dot{\tau}(t) R_{3}-\delta \dot{\tau}(t) R_{2}+R_{1}\right] \dot{x}(s) d s \\
& \leqslant\left[\begin{array}{c}
x(t) \\
x\left(t-\delta h_{a}\right)
\end{array}\right]^{\top}\left\{\left[\begin{array}{cc}
M_{4}+M_{4}^{\top} & -M_{4}+N_{4}^{\top} \\
* & -N_{4}-N_{4}^{\top}
\end{array}\right]+\delta h_{a} Z_{1}\right\}\left[\begin{array}{c}
x(t) \\
x\left(t-\delta h_{a}\right)
\end{array}\right] \\
& =\xi^{\top}(t)\left\{\left[\begin{array}{c}
e_{1} \\
e_{3}
\end{array}\right]^{\top}\left[\left[\begin{array}{cc}
M_{4}+M_{4}^{\top} & -M_{4}+N_{4}^{\top} \\
* & -N_{4}-N_{4}^{\top}
\end{array}\right]+\delta h_{a} Z_{1}\right]\left[\begin{array}{l}
e_{1} \\
e_{3}
\end{array}\right]\right\} \xi(t),
\end{aligned}
$$


with

$$
\left[\begin{array}{cc}
\dot{\tau}(t) R_{4}+(\delta-1) \dot{\tau}(t) R_{3}+(1-\delta \dot{\tau}(t)) R_{2} & {\left[\begin{array}{cc}
M_{3} & N_{3}
\end{array}\right]} \\
* & Z_{2}
\end{array}\right] \geqslant 0
$$

and

$$
\left[\begin{array}{cc}
\dot{\tau}(t) R_{4}+(\delta-1) \dot{\tau}(t) R_{3}-\delta \dot{\tau}(t) R_{2}+R_{1} & {\left[\begin{array}{cc}
M_{4} & N_{4} \\
Z_{1}
\end{array}\right]}
\end{array}\right] \geqslant 0,
$$

respectively.

Moreover, using Lemma 2.3 to (3.10) can yield the following inequality

$$
\begin{aligned}
& \dot{V}_{4}\left(t, x_{t}, \dot{x}_{t}\right)=\zeta_{0}^{T}\left[\tau_{0} S_{1}+\tau_{0} S_{2}+\tau_{s} S_{3}+\tau_{s} S_{4}+\bar{\tau}^{2} R_{s}\right] \zeta_{0}-\int_{-h_{a}}^{0} \int_{t+\theta}^{t} \dot{x}^{\top}(s) S_{1} \dot{x}(s) d s d \theta \\
& -\int_{-h_{a}}^{0} \int_{t-h_{a}}^{t+\theta} \dot{x}^{\top}(s) S_{2} \dot{x}(s) d s d \theta-\int_{-\tau(t)}^{-h_{a}} \int_{t+\theta}^{t-h_{a}} \dot{x}^{\top}(s) S_{3} \dot{x}(s) d s d \theta \\
& -\int_{-h_{b}}^{-\tau(t)} \int_{t+\theta}^{t-\tau(t)} \dot{x}^{\top}(s) S_{3} \dot{x}(s) d s d \theta-\left(h_{b}-\tau(t)\right) \int_{t-\tau(t)}^{t-h_{a}} \dot{x}^{\top}(s) S_{3} \dot{x}(s) d s \\
& -\int_{-\tau(t)}^{-h_{a}} \int_{t-\tau(t)}^{t+\theta} \dot{\chi}^{\top}(s) S_{4} \dot{x}(s) d s d \theta-\int_{-h_{b}}^{-\tau(t)} \int_{t-h_{b}}^{t+\theta} \dot{\chi}^{\top}(s) S_{4} \dot{x}(s) d s d \theta \\
& -\left(\tau(t)-h_{a}\right) \int_{t-h_{b}}^{t-\tau(t)} \dot{\chi}^{\top}(s) S_{4} \dot{x}(s) d s \\
& -\bar{\tau} \int_{t-\tau(t)}^{t-h_{a}} \dot{x}^{\top}(s) R_{s} \dot{x}(s) d s d \theta-\bar{\tau} \int_{t-h_{b}}^{t-\tau(t)} \dot{\chi}^{\top}(s) R_{s} \dot{x}(s) d s d \theta \\
& \leqslant \zeta_{0}^{\mathrm{T}}\left[\tau_{0} S_{1}+\tau_{0} S_{2}+\tau_{s} S_{3}+\tau_{s} S_{4}+\bar{\tau}^{2} R_{s}\right] \zeta_{0} \\
& -\xi^{\top}(t)\left\{2\left(e_{1}-e_{8}\right)^{\top} S_{1}\left(e_{1}-e_{8}\right)+4\left(e_{1}+2 e_{8}-3 e_{11}\right)^{\top} S_{1}\left(e_{1}+2 e_{8}-3 e_{11}\right)\right. \\
& +2\left(e_{8}-e_{5}\right)^{\top} S_{2}\left(e_{8}-e_{5}\right)+4\left(e_{5}-4 e_{8}+3 e_{11}\right)^{\top} S_{2}\left(e_{5}-4 e_{8}+3 e_{11}\right) \\
& +2\left(e_{5}-e_{9}\right)^{\top} S_{3}\left(e_{5}-e_{9}\right)+4\left(e_{5}+2 e_{9}-3 e_{12}\right)^{\top} S_{3}\left(e_{5}+2 e_{9}-3 e_{12}\right) \\
& +2\left(e_{2}-e_{10}\right)^{\top} S_{3}\left(e_{2}-e_{10}\right)+4\left(e_{2}+2 e_{10}-3 e_{13}\right)^{\top} S_{3}\left(e_{2}+2 e_{10}-3 e_{13}\right) \\
& +2\left(e_{9}-e_{2}\right)^{\mathrm{T}} S_{4}\left(e_{9}-e_{2}\right)+4\left(e_{2}-4 e_{9}+3 e_{12}\right)^{\top} S_{4}\left(e_{2}-4 e_{9}+3 e_{12}\right) \\
& +2\left(e_{10}-e_{6}\right)^{\mathrm{T}} S_{4}\left(e_{10}-e_{6}\right)+4\left(e_{6}-4 e_{10}+3 e_{13}\right)^{\mathrm{T}} S_{4}\left(e_{6}-4 e_{10}+3 e_{13}\right) \\
& +\left(\frac{\bar{\tau}}{\tau(t)-h_{a}}-1\right)\left[\left(e_{5}-e_{2}\right)^{\top} S_{3}\left(e_{5}-e_{2}\right)+3\left(e_{5}+e_{2}-2 e_{9}\right)^{\top} S_{3}\left(e_{5}+e_{2}-2 e_{9}\right)\right. \\
& \left.+5\left(e_{5}-e_{2}+6 e_{9}-6 e_{12}\right)^{\top} S_{3}\left(e_{5}-e_{2}+6 e_{9}-6 e_{12}\right)\right] \\
& +\left(\frac{\bar{\tau}}{h_{b}-\tau(t)}-1\right)\left[\left(e_{2}-e_{6}\right)^{\top} S_{4}\left(e_{2}-e_{6}\right)+3\left(e_{2}+e_{6}-2 e_{10}\right)^{\top} S_{4}\left(e_{2}+e_{6}-2 e_{10}\right)\right. \\
& \left.+5\left(e_{2}-e_{6}+6 e_{10}-6 e_{13}\right)^{\top} S_{4}\left(e_{2}-e_{6}+6 e_{10}-6 e_{13}\right)\right] \\
& +\frac{\bar{\tau}}{\tau(t)-h_{a}}\left[\left(e_{5}-e_{2}\right)^{\mathrm{T}} R_{s}\left(e_{5}-e_{2}\right)+3\left(e_{5}+e_{2}-2 e_{9}\right)^{\mathrm{T}} R_{s}\left(e_{5}+e_{2}-2 e_{9}\right)\right. \\
& \left.+5\left(e_{5}-e_{2}+6 e_{9}-6 e_{12}\right)^{\top} R_{s}\left(e_{5}-e_{2}+6 e_{9}-6 e_{12}\right)\right] \\
& +\frac{\bar{\tau}}{h_{b}-\tau(t)}\left[\left(e_{2}-e_{6}\right)^{\top} R_{s}\left(e_{2}-e_{6}\right)+3\left(e_{2}+e_{6}-2 e_{10}\right)^{\top} R_{s}\left(e_{2}+e_{6}-2 e_{10}\right)\right. \\
& \left.\left.+5\left(e_{2}-e_{6}+6 e_{10}-6 e_{13}\right)^{\mathrm{T}} R_{s}\left(e_{2}-e_{6}+6 e_{10}-6 e_{13}\right)\right]\right\} \xi(t) .
\end{aligned}
$$


Let us define $\alpha=\frac{\tau(t)-h_{a}}{\bar{\tau}}, \beta=\frac{h_{b}-\tau(t)}{\bar{\tau}}$. Employing the reciprocal convexity lemma in [16], we have

$$
\begin{aligned}
& -\xi^{\top}(t)\left\{\frac { \overline { \tau } } { h _ { b } - \tau ( t ) } \left[\left(e_{2}-e_{6}\right)^{\top} R_{s}\left(e_{2}-e_{6}\right)+3\left(e_{2}+e_{6}-2 e_{10}\right)^{\top} R_{s}\left(e_{2}+e_{6}-2 e_{10}\right)\right.\right. \\
& \left.+5\left(e_{2}-e_{6}+6 e_{10}-6 e_{13}\right)^{\mathrm{T}} R_{s}\left(e_{2}-e_{6}+6 e_{10}-6 e_{13}\right)\right] \\
& +\left(\frac{\bar{\tau}}{\tau(\mathrm{t})-h_{a}}-1\right)\left[\left(e_{5}-e_{2}\right)^{\top} S_{3}\left(e_{5}-e_{2}\right)+3\left(e_{5}+e_{2}-2 e_{9}\right)^{\top} S_{3}\left(e_{5}+e_{2}-2 e_{9}\right)\right. \\
& \left.+5\left(e_{5}-e_{2}+6 e_{9}-6 e_{12}\right)^{\top} S_{3}\left(e_{5}-e_{2}+6 e_{9}-6 e_{12}\right)\right] \\
& +\left(\frac{\bar{\tau}}{h_{b}-\tau(t)}-1\right)\left[\left(e_{2}-e_{6}\right)^{\top} S_{4}\left(e_{2}-e_{6}\right)+3\left(e_{2}+e_{6}-2 e_{10}\right)^{\top} S_{4}\left(e_{2}+e_{6}-2 e_{10}\right)\right. \\
& \left.+5\left(e_{2}-e_{6}+6 e_{10}-6 e_{13}\right)^{\top} S_{4}\left(e_{2}-e_{6}+6 e_{10}-6 e_{13}\right)\right] \\
& +\frac{\bar{\tau}}{\tau(t)-h_{a}}\left[\left(e_{5}-e_{2}\right)^{\top} R_{s}\left(e_{5}-e_{2}\right)+3\left(e_{5}+e_{2}-2 e_{9}\right)^{\top} R_{s}\left(e_{5}+e_{2}-2 e_{9}\right)\right. \\
& \left.\left.+5\left(e_{5}-e_{2}+6 e_{9}-6 e_{12}\right)^{\mathrm{T}} R_{s}\left(e_{5}-e_{2}+6 e_{9}-6 e_{12}\right)\right]\right\} \xi(t) \\
& =-\frac{1}{\alpha} \xi^{\top}(t)\left\{\begin{array}{l}
\left(e_{5}-e_{2}\right)^{\top}\left(R_{s}+S_{3}\right)\left(e_{5}-e_{2}\right)+3\left(e_{5}+e_{2}-2 e_{9}\right)^{\top}\left(R_{s}+S_{3}\right)\left(e_{5}+e_{2}-2 e_{9}\right) \\
+5\left(e_{5}-e_{2}+6 e_{9}-6 e_{12}\right)^{\top}\left(R_{s}+S_{3}\right)\left(e_{5}-e_{2}+6 e_{9}-6 e_{12}\right)
\end{array}\right\} \xi(t) \\
& -\frac{1}{\beta} \xi^{\top}(t)\left\{\begin{array}{l}
\left(e_{2}-e_{6}\right)^{\top}\left(R_{s}+S_{4}\right)\left(e_{2}-e_{6}\right)+3\left(e_{2}+e_{6}-2 e_{10}\right)^{\top}\left(R_{s}+S_{4}\right)\left(e_{2}+e_{6}-2 e_{10}\right) \\
+5\left(e_{2}-e_{6}+6 e_{10}-6 e_{13}\right)^{\top}\left(R_{s}+S_{4}\right)\left(e_{2}-e_{6}+6 e_{10}-6 e_{13}\right)
\end{array}\right\} \xi(t) \\
& +\xi^{\top}(t)\left\{\begin{array}{l}
\left(e_{5}-e_{2}\right)^{\top} S_{3}\left(e_{5}-e_{2}\right)+3\left(e_{5}+e_{2}-2 e_{9}\right)^{\top} S_{3}\left(e_{5}+e_{2}-2 e_{9}\right) \\
+5\left(e_{5}-e_{2}+6 e_{9}-6 e_{12}\right)^{\top} S_{3}\left(e_{5}-e_{2}+6 e_{9}-6 e_{12}\right)+ \\
\left(e_{2}-e_{6}\right)^{\top} S_{4}\left(e_{2}-e_{6}\right)+3\left(e_{2}+e_{6}-2 e_{10}\right)^{\top} S_{4}\left(e_{2}+e_{6}-2 e_{10}\right) \\
+5\left(e_{2}-e_{6}+6 e_{10}-6 e_{13}\right)^{\top} S_{4}\left(e_{2}-e_{6}+6 e_{10}-6 e_{13}\right)
\end{array}\right\} \xi(t) \\
& \leqslant-\xi^{\top}(\mathrm{t}) \Psi \xi(\mathrm{t}) \text {, }
\end{aligned}
$$

It follows from (3.7)-(3.16) that:

$$
\dot{V}\left(t, x_{t}, \dot{x}_{t}\right) \leqslant \xi^{\top}(t)[\Xi(\tau(t), \dot{\tau}(t))] \xi(t),
$$

where

$$
\begin{aligned}
& \bar{\Xi}(\tau(t), \dot{\tau}(t))=\bar{\zeta}_{0}^{\top}\left(\Xi_{00}+P_{\delta}+\tau_{0} S_{1}+\tau_{0} S_{2}+\tau_{s} S_{3}+\tau_{s} S_{4}+\bar{\tau}^{2} R_{s}\right) \bar{\zeta}_{0}+\zeta_{1}^{\top} \mathrm{P}(\tau(t)) \zeta_{2}+\zeta_{2}^{\top} \mathrm{P}(\tau(\mathrm{t})) \zeta_{1} \\
& +(1-\delta \dot{\tau}(\mathrm{t}))\left[\zeta_{1}^{\top} \mathrm{P}(\tau(\mathrm{t})) \zeta_{3}+\zeta_{3}^{\mathrm{T}} \mathrm{P}(\tau(\mathrm{t})) \zeta_{1}-e_{7}^{\mathrm{T}} \mathrm{P}_{\delta} \mathrm{e}_{7}\right]+\zeta_{1}^{\mathrm{T}}\left[\frac{\dot{\tau}(\mathrm{t})}{\bar{\tau}}\left(\mathrm{P}^{1}-\mathrm{P}^{2}\right)\right] \zeta_{1} \\
& -\left\{2\left(e_{1}-e_{8}\right)^{\top} S_{1}\left(e_{1}-e_{8}\right)+4\left(e_{5}+2 e_{8}-3 e_{11}\right)^{\top} S_{1}\left(e_{1}+2 e_{8}-3 e_{11}\right)\right. \\
& +2\left(e_{8}-e_{5}\right)^{\top} S_{2}\left(e_{8}-e_{5}\right)+4\left(e_{5}-4 e_{8}+3 e_{11}\right)^{\top} S_{2}\left(e_{5}-4 e_{8}+3 e_{11}\right) \\
& +2\left(e_{5}-e_{9}\right)^{\mathrm{T}} S_{3}\left(e_{5}-e_{9}\right)+4\left(e_{5}+2 e_{9}-3 e_{12}\right)^{\mathrm{T}} S_{3}\left(e_{5}+2 e_{9}-3 e_{12}\right) \\
& +2\left(e_{2}-e_{10}\right)^{\mathrm{T}} S_{3}\left(e_{2}-e_{10}\right)+4\left(e_{2}+2 e_{10}-3 e_{13}\right)^{\mathrm{T}} S_{3}\left(e_{2}+2 e_{10}-3 e_{13}\right) \\
& +2\left(e_{9}-e_{2}\right)^{\mathrm{T}} S_{4}\left(e_{9}-e_{2}\right)+4\left(e_{2}-4 e_{9}+3 e_{12}\right)^{\mathrm{T}} S_{4}\left(e_{2}-4 e_{9}+3 e_{12}\right) \\
& \left.+2\left(e_{10}-e_{6}\right)^{\top} S_{4}\left(e_{10}-e_{6}\right)+4\left(e_{6}-4 e_{10}+3 e_{13}\right)^{\top} S_{4}\left(e_{6}-4 e_{10}+3 e_{13}\right)\right\} \\
& +e_{1}^{\top} \mathrm{Q}_{1} e_{1}+e_{3}^{\top}\left(\mathrm{Q}_{2}-\mathrm{Q}_{1}\right) e_{3}+(1-\delta \dot{\tau}(\mathrm{t})) e_{4}^{\top}\left(\mathrm{Q}_{3}-\mathrm{Q}_{2}\right) e_{4}+(1-\dot{\tau}(\mathrm{t})) e_{2}^{\top}\left(\mathrm{Q}_{4}-\mathrm{Q}_{3}\right) e_{2}-e_{5}^{\top} \mathrm{Q}_{4} e_{5} \\
& +\left[\begin{array}{l}
e_{2} \\
e_{6}
\end{array}\right]^{\top}\left\{M N_{1}+\left(h_{b}-\tau(t)\right)\left[\begin{array}{l}
M_{1} \\
N_{1}
\end{array}\right] R_{4}^{-1}\left[\begin{array}{ll}
M_{1}^{\top} & N_{1}^{\top}
\end{array}\right]\right\}\left[\begin{array}{l}
e_{2} \\
e_{6}
\end{array}\right] \\
& +\left[\begin{array}{l}
e_{4} \\
e_{2}
\end{array}\right]^{\top}\left\{M N_{2}+(1-\delta) \tau(t) Z_{3}\right\}\left[\begin{array}{l}
e_{4} \\
e_{2}
\end{array}\right]+\left[\begin{array}{l}
e_{3} \\
e_{4}
\end{array}\right]^{\top}\left\{M N_{3}+\delta\left(\tau(t)-h_{a}\right) Z_{2}\right\}\left[\begin{array}{l}
e_{3} \\
e_{4}
\end{array}\right]
\end{aligned}
$$




$$
+\left[\begin{array}{l}
e_{1} \\
e_{3}
\end{array}\right]^{\top}\left\{\mathrm{MN}_{4}+\delta h_{\mathrm{a}} \mathrm{Z}_{1}\right\}\left[\begin{array}{l}
e_{1} \\
e_{3}
\end{array}\right]-\Psi
$$

and $\bar{\zeta}_{0}=A(t) e_{1}+A_{\tau}(t) e_{2}$, and the other denotations are defined as (3.5), with (3.2), (3.3), (3.4) hold.

In addition, if $\bar{\Xi}(\tau(t), \dot{\tau}(t))<0$, then $\bar{\Xi}_{k}(\tau(t), \dot{\tau}(t))=\left.\bar{\Xi}(\tau(t), \dot{\tau}(t))\right|_{\dot{\tau}(t)=d_{k}}<0,(k=1,2)$.

Note that $\bar{\Xi}(\tau(t), \dot{\tau}(t))=\frac{d_{2}-\dot{\tau}(t)}{d_{2}-d_{1}} \bar{\Xi}_{1}(\tau(t), \dot{\tau}(t))+\frac{\dot{\tau}(t)-d_{1}}{d_{2}-d_{1}} \bar{\Xi}_{2}(\tau(t), \dot{\tau}(t))$, and then $\bar{\Xi}(\tau(t), \dot{\tau}(t))$ are convex in $\dot{\tau}(\mathrm{t}) \in\left[\mathrm{d}_{1}, \mathrm{~d}_{2}\right]$.

So far, we can conclude that asymptotically stability of the T-S fuzzy system (2.2) is guaranteed if

$$
\Xi\left(\tau(t), d_{1}\right)<0 \text { and } \Xi\left(\tau(t), d_{2}\right)<0 .
$$

Since this uncertain parameter appears linearly in (3.18), the uncertain set can be described by a polytope [8]. The vertices of this set can be calculated by setting the parameter to either lower or upper limit. Then, for given $0<h_{a} \leqslant \tau(t) \leqslant h_{b}$, since $d_{1} \leqslant \dot{\tau}(t) \leqslant d_{2}, \bar{\Xi}(\tau(t), \dot{\tau}(t))<0$ holds if (3.18) is true, and then (3.17) is true. Furthermore, following [9], based on convex combination method, for T-S fuzzy system in the form of (2.2), (3.1) implies $\sum_{l=1}^{r} h_{l}(\theta(t)) \Xi_{l}\left(\tau(t), d_{1}\right)<0$ and $\sum_{l=1}^{r} h_{l}(\theta(t)) \Xi_{l}\left(\tau(t), d_{2}\right)<0$, which is equivalent to (3.17). By using Theorem 3.1 in [9], from the above process, if the LMIs in (3.1) are feasible, the T-S fuzzy system (2.2) is asymptotically stable. This completes the proof.

Remark 3.2. Unlike the complete Lyapunov functional method in $[7,10]$ and the delay partitioning technique in [11,32], in this paper a Lyapunov-Krasovskii functional containing the variable delay-partitioning tuning parameter $0<\delta<1$ is constructed, which means that different delay-partitioning tuning parameter $0<\delta<1$ can obtain different LMI-based stability condition. Our derivation towards Theorem 3.1 also adopts some newly developed integral inequalities (Lemma 2.3) in [23] while the present result is derived by some appropriate integral inequalities in Lemma 2.2. The key is that the double integral inequalities in Lemma 2.3 give much tighter upper bounds in Eqs. (3.15) and (3.16) than those obtained by Jensen inequality. Therefore, the resulting stability criterion in Theorem 3.1 is much less conservative than the ones based on Jensen inequality.

Remark 3.3. It is worth pointing out that in the case that $\mathrm{d}_{2}<1$, it is clear that less conservative stability criteria can be derived by introducing the term $\int_{t-\tau(t)}^{t-h_{1}} \chi^{\top}(s) Q_{\tau} \chi(s) d s$ with $h_{1} \leqslant \tau(t) \leqslant h_{2}$ in the existing literatures. However, in the case that $d_{2} \geqslant 1.0$, it can be seen from $[2,10,12,13,18,20-22,25,27,30,32,34]$ that this term has no help for deriving less conservative stability criteria while the present results can work.

The utilized state-augmented vector $\xi(t)$ includes newly proposed double integral terms such as $\frac{2}{h_{a}^{2}} \int_{-h_{a}}^{0} \int_{t+\theta}^{t} \chi(s) d s d \theta, \frac{2}{\left(\tau(t)-h_{a}\right)^{2}} \int_{-\tau(t)}^{-h_{a}} \int_{t+\theta}^{t}-h_{a} x(s) d s d \theta, \frac{2}{\left(h_{b}-\tau(t)\right)^{2}} \int_{-h_{b}}^{-\tau(t)} \int_{t+\theta}^{t-\tau(t)} \chi(s) d s d \theta$. In addition, we introduced the integral terms with variable delay-partitioning tuning parameter $\delta$ satisfying $\delta d_{2}<1$ such as $\left[\begin{array}{c}x(t) \\ x(t-\delta \tau(t))\end{array}\right]^{\top} P(\tau(t))\left[\begin{array}{c}x(t) \\ x(t-\delta \tau(t))\end{array}\right], \int_{t-\tau(t)}^{t-\delta \tau(t)} x^{\top}(s) Q_{3} x(s) d s, \int_{t-\delta \tau(t)}^{t} \dot{x}^{\top}(s) P_{\delta} \dot{x}(s) d s$. All the resulting relations in (3.6) are well employed and the stability criterion is given in the form of LMIs. Such a feature leads to less conservative results compared to the existing ones.

When we set $\mathrm{Q}_{1}=\mathrm{Q}_{2}=\mathrm{Q}_{3}=\mathrm{Q}_{4}=\mathrm{Q}$ and $\mathrm{R}_{1}=\mathrm{R}_{2}=\mathrm{R}_{3}=\mathrm{R}_{4}=\mathrm{R}$, furthermore, we set $\mathrm{P}^{1}=\mathrm{P}^{2}$, and $\mathrm{P}_{\mathrm{i} 2}=0, \mathrm{P}_{\mathrm{i} 3}=0$, Theorem 3.1 reduces to the following corollary.

Corollary 3.4. For given scalars $0<\mathrm{h}_{\mathrm{a}} \leqslant \mathrm{h}_{\mathrm{b}}, \mathrm{d}_{1} \leqslant \mathrm{~d}_{2}$, and $0<\delta<1$, satisfying $\delta \mathrm{d}_{2}<1$, the T-S fuzzy system (2.2) is asymptotically stable if there exist real symmetry matrices $P_{1}>0, P_{\delta}>0, Q_{j}=Q>0, R_{j}=R>0$, $S_{j}>0,(j=1,2,3,4), R_{s}>0$, and any matrices $\Theta=\left[\Theta_{i j}\right]_{3 \times 3^{\prime}} Z_{j}=\left[\begin{array}{cc}Z_{j 1} & Z_{j 2} \\ * & Z_{j 3}\end{array}\right],(j=1,2,3), M_{i}, N_{i}$, $(i=1,2,3,4)$ with appropriate dimensions such that the LMIs in (3.19) when $\tau(t) \rightarrow h_{a}$ and $\tau(t) \rightarrow h_{b}$ hold, for any $l=1,2, \cdots, r$.

$$
\tilde{\Xi}_{l}\left(\tau(t), d_{1}\right)<0 \text { and } \tilde{\Xi}_{l}\left(\tau(t), d_{2}\right)<0
$$


with (3.2), (3.3), (3.4) hold, and

$$
\left[\begin{array}{cc}
\mathrm{RS}_{3} & \Theta \\
* & \mathrm{RS}_{4}
\end{array}\right]>0
$$

where the denotations are defined in (3.5) and

$$
\begin{aligned}
\tilde{\Xi}_{l}(\tau(t), \dot{\tau}(t))= & \zeta_{0}^{\top} \Xi_{0} \zeta_{0}+\zeta_{1}^{\top} P_{1} \zeta_{2}+\zeta_{2}^{\top} P_{1} \zeta_{1}-(1-\delta \dot{\tau}(t))\left[e_{7}^{\top} P_{\delta} e_{7}\right] \\
& -\left\{2\left(e_{1}-e_{8}\right)^{\top} S_{1}\left(e_{1}-e_{8}\right)+4\left(e_{1}+2 e_{8}-3 e_{11}\right)^{\top} S_{1}\left(e_{1}+2 e_{8}-3 e_{11}\right)\right. \\
& +2\left(e_{8}-e_{5}\right)^{\top} S_{2}\left(e_{8}-e_{5}\right)+4\left(e_{5}-4 e_{8}+3 e_{11}\right)^{\top} S_{2}\left(e_{5}-4 e_{8}+3 e_{11}\right) \\
& +2\left(e_{5}-e_{9}\right)^{\top} S_{3}\left(e_{5}-e_{9}\right)+4\left(e_{5}+2 e_{9}-3 e_{12}\right)^{\top} S_{3}\left(e_{5}+2 e_{9}-3 e_{12}\right) \\
& +2\left(e_{2}-e_{10}\right)^{\top} S_{3}\left(e_{2}-e_{10}\right)+4\left(e_{2}+2 e_{10}-3 e_{13}\right)^{\top} S_{3}\left(e_{2}+2 e_{10}-3 e_{13}\right) \\
& +2\left(e_{9}-e_{2}\right)^{\top} S_{4}\left(e_{9}-e_{2}\right)+4\left(e_{2}-4 e_{9}+3 e_{12}\right)^{\top} S_{4}\left(e_{2}-4 e_{9}+3 e_{12}\right) \\
& \left.+2\left(e_{10}-e_{6}\right)^{\top} S_{4}\left(e_{10}-e_{6}\right)+4\left(e_{6}-4 e_{10}+3 e_{13}\right)^{\top} S_{4}\left(e_{6}-4 e_{10}+3 e_{13}\right)\right\} \\
& +e_{1}^{\top} Q_{1} e_{1}+e_{3}^{\top}\left(Q_{2}-Q_{1}\right) e_{3}+(1-\delta \dot{\tau}(t)) e_{4}^{\top}\left(Q_{3}-Q_{2}\right) e_{4}+(1-\dot{\tau}(t)) e_{2}^{\top}\left(Q_{4}-Q_{3}\right) e_{2}-e_{5}^{T} Q_{4} e_{5} \\
& +\left[\begin{array}{c}
e_{2} \\
e_{6}
\end{array}\right]^{\top}\left\{M N_{1}+\left(h_{b}-\tau(t)\right)\left[\begin{array}{c}
M \\
N_{1}
\end{array}\right] R_{4}^{-1}\left[M_{1}^{\top} N_{1}^{\top}\right]\right\}\left[\begin{array}{c}
e_{2} \\
e_{6}
\end{array}\right] \\
& +\left[\begin{array}{c}
e_{4} \\
e_{2}
\end{array}\right]^{\top}\left\{M N_{2}+(1-\delta) \tau(t) Z_{3}\right\}\left[\begin{array}{c}
e_{4} \\
e_{2}
\end{array}\right]+\left[\begin{array}{c}
e_{3} \\
e_{4}
\end{array}\right]^{\top}\left\{M N_{3}+\delta\left(\tau(t)-h_{a}\right) Z_{2}\right\}\left[\begin{array}{c}
e_{3} \\
e_{4}
\end{array}\right] \\
& +\left[\begin{array}{c}
e_{1} \\
e_{3}
\end{array}\right]^{\top}\left\{M N_{4}+\delta h_{a} Z_{1}\right\}\left[\begin{array}{c}
e_{1} \\
e_{3}
\end{array}\right]-\Psi,
\end{aligned}
$$

when the lower bound of the delay is 0 , that is, $\mathrm{h}_{\mathrm{a}}=0$, the interval team $\left[0, \delta \mathrm{h}_{\mathrm{a}}\right]$ is missing, and for simplicity we denote

$$
\begin{aligned}
& \hat{\xi}(t):=\operatorname{col}\left\{x(t) \quad x(t-\tau(t)) \quad x(t-\delta \tau(t)) \quad x\left(t-h_{b}\right) \quad \dot{x}(t-\delta \tau(t)) \quad \hat{\xi}_{0}(t)\right\} \\
& \hat{\xi}_{0}(t):=\operatorname{col}\left\{\frac{1}{\tau(t)} \int_{t-\tau(t)}^{t} x(s) d s \quad \frac{1}{h_{b}-\tau(t)} \int_{t-h_{b}}^{t-\tau(t)} x(s) d s \quad \frac{2}{(\tau(t))^{2}} \int_{-\tau(t)}^{0} \int_{t+\theta}^{t} x(s) d s d \theta\right. \\
& \left.\frac{2}{\left(h_{b}-\tau(t)\right)^{2}} \int_{-h_{b}}^{-\tau(t)} \int_{t+\theta}^{t-\tau(t)} x(s) d s d \theta\right\}
\end{aligned}
$$

and $\hat{\mathrm{e}}_{i},(\mathrm{i}=1,2, \cdots, 9)$ are block entry matrices, for example, $\hat{\mathrm{e}}_{1}=\left[\begin{array}{ccccccccc}\mathrm{I} & 0 & 0 & 0 & 0 & 0 & 0 & 0 & 0\end{array}\right]$.

Theorem 3.5. For given scalars $0=\mathrm{h}_{\mathrm{a}} \leqslant \mathrm{h}_{\mathrm{b}}, \mathrm{d}_{1} \leqslant \mathrm{~d}_{2}$, and $0<\delta<1$, satisfying $\delta \mathrm{d}_{2}<1$, the T-S fuzzy system (2.2) is asymptotically stable if there exist real symmetry matrices $\mathrm{P}^{\mathrm{i}}=\left[\begin{array}{cc}\mathrm{P}_{\mathrm{i} 1} & \mathrm{P}_{\mathrm{i} 2} \\ * & \mathrm{P}_{\mathrm{i} 3}\end{array}\right] \geqslant 0$ with $\mathrm{P}_{\mathrm{i} 1}>0$, $(i=1,2), P_{\delta}>0, Q_{j}>0, R_{j}>0,(j=2,3,4), S_{3}>0, S_{4}>0, R_{s}>0$, and any matrices $\Theta=\left[\Theta_{i j}\right]_{3 \times 3^{\prime}}$ $Z_{j}=\left[\begin{array}{cc}Z_{j 1} & Z_{j 2} \\ * & Z_{j 3}\end{array}\right],(j=2,3), M_{i}, N_{i},(i=1,2,3)$ with appropriate dimensions such that the LMIs in (3.20) when $\tau(t) \rightarrow h_{a}$ and $\tau(t) \rightarrow h_{b}$ hold, for any $l=1,2, \cdots, r$.

$$
\hat{\Xi}_{l}\left(\tau(\mathrm{t}), \mathrm{d}_{1}\right)<0 \text { and } \hat{\Xi}_{l}\left(\tau(\mathrm{t}), \mathrm{d}_{2}\right)<0
$$

with (3.2) and (3.3) hold, and

$$
\left[\begin{array}{cc}
\mathrm{RS}_{3} & \Theta \\
* & \mathrm{RS}_{4}
\end{array}\right]>0
$$


where

$$
\begin{aligned}
& \hat{\Xi}_{l}(\tau(t), \dot{\tau}(t))=\hat{\zeta}_{0}^{\top} \hat{\Xi}_{0} \hat{\zeta}_{0}+\hat{\zeta}_{1}^{\top} P(\tau(t)) \hat{\zeta}_{2}+\hat{\zeta}_{2}^{\top} P(\tau(t)) \hat{\zeta}_{1} \\
& +(1-\delta \dot{\tau}(\mathrm{t}))\left[\hat{\zeta}_{1}^{\mathrm{T}} \mathrm{P}(\tau(\mathrm{t})) \hat{\zeta}_{3}+\hat{\zeta}_{3}^{\mathrm{T}} \mathrm{P}(\tau(\mathrm{t})) \hat{\zeta}_{1}-\hat{e}_{5}^{\mathrm{T}} \mathrm{P}_{\delta} \hat{e}_{5}\right]+\hat{\zeta}_{1}^{\mathrm{T}}\left[\frac{\dot{\tau}(\mathrm{t})}{\bar{\tau}}\left(\mathrm{P}^{1}-\mathrm{P}^{2}\right)\right] \hat{\zeta}_{1} \\
& -\left\{2\left(e_{1}-e_{6}\right)^{\top} S_{3}\left(e_{1}-e_{6}\right)+4\left(e_{1}+2 e_{6}-3 e_{8}\right)^{\top} S_{3}\left(e_{1}+2 e_{6}-3 e_{8}\right)\right. \\
& +2\left(e_{2}-e_{7}\right)^{\top} S_{3}\left(e_{2}-e_{7}\right)+4\left(e_{2}+2 e_{7}-3 e_{9}\right)^{\top} S_{3}\left(e_{2}+2 e_{7}-3 e_{9}\right) \\
& +2\left(e_{6}-e_{2}\right)^{\top} S_{4}\left(e_{6}-e_{2}\right)+4\left(e_{2}-4 e_{6}+3 e_{8}\right)^{\top} S_{4}\left(e_{2}-4 e_{6}+3 e_{8}\right) \\
& \left.+2\left(e_{7}-e_{4}\right)^{\top} S_{4}\left(e_{7}-e_{4}\right)+4\left(e_{4}-4 e_{7}+3 e_{9}\right)^{\top} S_{4}\left(e_{4}-4 e_{7}+3 e_{9}\right)\right\} \\
& +\hat{e}_{1}^{\top}\left(Q_{2}\right) \hat{e}_{1}+(1-\delta \dot{\tau}(t)) \hat{e}_{3}^{\top}\left(Q_{3}-Q_{2}\right) \hat{e}_{3}+(1-\dot{\tau}(t)) \hat{e}_{2}^{\top}\left(Q_{4}-Q_{3}\right) \hat{e}_{2}-\hat{e}_{4}^{\top} Q_{4} \hat{e}_{4} \\
& +\left[\begin{array}{l}
\hat{e}_{2} \\
\hat{e}_{4}
\end{array}\right]^{\top}\left\{M N_{1}+\left(h_{b}-\tau(t)\right)\left[\begin{array}{c}
M_{1} \\
N_{1}
\end{array}\right] R_{4}^{-1}\left[\begin{array}{ll}
M_{1}^{\top} & N_{1}^{\top}
\end{array}\right]\right\}\left[\begin{array}{l}
\hat{e}_{2} \\
\hat{e}_{4}
\end{array}\right] \\
& +\left[\begin{array}{l}
\hat{e}_{3} \\
\hat{e}_{2}
\end{array}\right]^{\top}\left\{M N_{2}+(1-\delta) \tau(t) Z_{3}\right\}\left[\begin{array}{l}
\hat{e}_{3} \\
\hat{e}_{2}
\end{array}\right] \\
& +\left[\begin{array}{l}
\hat{e}_{1} \\
\hat{e}_{4}
\end{array}\right]^{\top}\left\{M N_{3}+\delta\left(\tau(t)-h_{a}\right) Z_{2}\right\}\left[\begin{array}{l}
\hat{e}_{1} \\
\hat{e}_{4}
\end{array}\right]-\hat{\Psi}, \\
& \hat{\Psi}=\hat{\Gamma}\left[\begin{array}{cc}
\operatorname{diag}\left\{R_{s}, 3 R_{s}, 5 R_{s}\right\} & \Theta \\
* & \operatorname{diag}\left\{R_{s}, 3 R_{s}, 5 R_{s}\right\}
\end{array}\right] \hat{\Gamma}^{\top}, \\
& \hat{\Gamma}=\left[\begin{array}{lll}
\left(e_{1}-e_{2}\right)^{\top} & \left(e_{1}+e_{2}-2 e_{6}\right)^{\top} & \left(e_{1}-e_{2}+6 e_{6}-6 e_{8}\right.
\end{array}\right)^{\top} \\
& \left.\times\left(e_{2}-e_{4}\right)^{\top} \quad\left(e_{2}+e_{4}-2 e_{7}\right)^{\top} \quad\left(e_{2}-e_{4}+6 e_{7}-6 e_{9}\right)^{\top}\right], \\
& R_{3}=\operatorname{diag}\left\{\begin{array}{lll}
R_{s}+S_{3} & 3\left(R_{s}+S_{3}\right) & 5\left(R_{s}+S_{3}\right)
\end{array}\right\}, \\
& R_{4}=\operatorname{diag}\left\{\begin{array}{lll}
R_{\mathrm{S}}+\mathrm{S}_{4} & 3\left(\mathrm{R}_{\mathrm{s}}+\mathrm{S}_{4}\right) & 5\left(\mathrm{R}_{\mathrm{s}}+\mathrm{S}_{4}\right)
\end{array}\right\} \text {, } \\
& \hat{\Xi}_{0}:=P_{\delta}+\delta\left(\tau(t)-h_{a}\right) R_{2}+(1-\delta) \tau(t) R_{3}+\left(h_{b}-\tau(t)\right) R_{4}+\tau_{s} S_{3}+\tau_{s} S_{4}+\bar{\tau}^{2} R_{s} \text {, } \\
& \hat{\zeta}_{0}=A_{l} \hat{e}_{1}+A_{\tau l} \hat{e}_{2} \hat{\zeta}_{1}=\left(\begin{array}{c}
\hat{e}_{1} \\
\hat{e}_{3}
\end{array}\right), \quad \hat{\zeta}_{2}=\left(\begin{array}{c}
\hat{\zeta}_{0} \\
0
\end{array}\right), \hat{\zeta}_{3}=\left(\begin{array}{c}
0 \\
\hat{e}_{5}
\end{array}\right) \text {. }
\end{aligned}
$$

Proof. Choose the following delay-dependent LK functional candidate as

$$
\hat{V}\left(t, x_{t}, \dot{x}_{t}\right)=V_{1}\left(t, x_{t}, \dot{x}_{t}\right)+\hat{V}_{2}\left(t, x_{t}, \dot{x}_{t}\right)+\hat{V}_{3}\left(t, x_{t}, \dot{x}_{t}\right)+\hat{V}_{4}\left(t, x_{t}, \dot{x}_{t}\right),
$$

where

$$
\begin{aligned}
\hat{V}_{2}\left(t, x_{t}, \dot{x}_{t}\right)= & \int_{t-\delta \tau(t)}^{t} x^{\top}(s) Q_{2} x(s) d s+\int_{t-\tau(t)}^{t-\delta \tau(t)} x^{\top}(s) Q_{3} x(s) d s+\int_{t-h_{b}}^{t-\tau(t)} x^{\top}(s) Q_{4} x(s) d s \\
\hat{V}_{3}\left(t, x_{t}, \dot{x}_{t}\right)= & \int_{-\delta \tau(t)}^{0} \int_{t+\theta}^{t} \dot{x}^{\top}(s) R_{2} \dot{x}(s) d s d \theta+\int_{-\tau(t)}^{-\delta \tau(t)} \int_{t+\theta}^{t} \dot{x}^{\top}(s) R_{3} \dot{x}(s) d s d \theta+\int_{-h_{b}}^{-\tau(t)} \int_{t+\theta}^{t} \dot{x}^{\top}(s) R_{4} \dot{x}(s) d s d \theta \\
\hat{V}_{4}\left(t, x_{t}, \dot{x}_{t}\right)= & \int_{-h_{b}}^{0} \int_{\theta}^{0} \int_{t+\lambda}^{t} \dot{x}^{\top}(s) S_{3} \dot{x}(s) d s d \lambda d \theta+\int_{-h_{b}}^{0} \int_{-h_{b}}^{\theta} \int_{t+\lambda}^{t} \dot{x}^{\top}(s) S_{4} \dot{x}(s) d s d \lambda d \theta \\
& +\bar{\tau} \int_{-h_{b}}^{0} \int_{t+\theta}^{t} \dot{x}^{\top}(s) R_{s} \dot{x}(s) d s d \theta .
\end{aligned}
$$

Then following the similar argument in the proof of Theorem 3.1, we can obtain the results. 
Remark 3.6. Theorems 3.1 and 3.5 depend on the parameter $0<\delta<1$ satisfying $\delta \mathrm{d}_{2}<1$. Such variable decomposition method may work when $\mathrm{d}_{2} \geqslant 1$, and then lead to reduction of conservatism if a suitable dividing point with relation to $\delta$ can be set. For seeking an appropriate $\delta$ satisfying $0<\delta<1$ and $\delta d_{2}<1$, we use fminsearch in the Optimization Toolbox of MATLAB, and one can refer to [33].

Remark 3.7. It should be noted that the utilized Lyapunov-Krasovskii functional in Theorems 3.1 and 3.5 is identical/similar to that of $[2,32,33]$. The main difference is that in $[2,32,33]$ the upper bounds of the quadratic integral terms are estimated by (improved) Jensen inequality, while in Theorems 3.1 and 3.5 they are conducted by the inequalities (3.11)-(3.14) which are derived from Lemma 2.2 and are estimated by the inequalities (3.15) and (3.16), which are derived from Lemma 2.3. Meanwhile, they are not introduced by some additional slack variables based on the Zero inequality, such as Newton-Leibniz formula. The results from two approaches will be compared in the next section.

\section{Numerical examples}

This section gives three examples to demonstrate the effectiveness and reduced conservatism of the proposed approach. For comparisons, we study T-S fuzzy system (2.2) with fuzzy rules investigated in recent publications $[1,2,10,12,13,20,21,25,27,32,33]$.

Example 4.1 (Example 1 of [13]). Consider a two rule system (2.2) with the following parameters:

$$
A_{1}=\left[\begin{array}{cc}
-2.1 & 0.1 \\
-0.2 & -0.9
\end{array}\right], A_{2}=\left[\begin{array}{cc}
-1.9 & 0 \\
-0.2 & -1.1
\end{array}\right], A_{\tau 1}=\left[\begin{array}{cc}
-1.1 & 0.1 \\
-0.8 & -0.9
\end{array}\right], A_{\tau 2}=\left[\begin{array}{cc}
-0.9 & 0 \\
-1.1 & -1.2
\end{array}\right],
$$

and, $h_{1}=\frac{1}{1+\exp \left(-2 x_{1}(t)\right)}, h_{2}=1-h_{1}$.

The purpose is to compare the maximum allowable delay bounds (MADB) of $\tau(t)$ that guarantees the asymptotic stability of the above system. To compare with the existing results in $[1,13$, 32], we assume that $h_{a}=0$. For given $d_{2}$ or mu, Table 1 gives the MADB on $h_{b}$ obtained by various methods in $[1,13,32]$ and this paper. From Table 1 , it can be seen that when the delay is time-invariant, i.e., $d_{2}=0$, the obtained results in Theorem 3.5 are better than those in $[1,13]$, while Theorem 3.5 can be complementary with the results in Zeng et al. [32]. Furthermore, only the conditions provided in Zeng et al. [32] are less conservative than the ones of Theorem 3.5 for slow varying delays. However for fast varying delays, Theorem 3.5 becomes less conservative than the conditions from these articles. Meanwhile, it can also be seen that the tuning parameter $\delta$ may have a key effect on the reduced conservatism of the proposed results, and different delay decomposition parameter and different lower bound of delay derivative may yield different LMI, and then lead different MADB on $h_{b}$ for guaranteeing the above system asymptotically stable.

Table 1: MADB on $h_{b}$ for various $d_{2}\left(h_{a}=0\right)$ for Example 4.1.

\begin{tabular}{lllll}
\hline Method & $\mathrm{d}_{2}=0.0$ & $\mathrm{~d}_{2}=0.1$ & $\mathrm{~d}_{2}=0.5$ & $\mathrm{~d}_{2}=1.1$ \\
\hline Liu et al. [13] & 3.290 & 2.710 & 1.750 & 1.440 \\
An et al. [1] $\left(\mathrm{d}_{1}=0\right)$ & 3.765 & 3.023 & 1.784 & 1.450 \\
Zeng et al. [32] & 4.290 & 3.350 & 1.930 & 1.710 \\
Theorem 3.1 $\left(\mathrm{d}_{1}=0\right.$ and $\left.\delta=0.8\right)$ & 4.023 & 3.532 & 2.355 & 1.897 \\
Theorem 3.5 $\left(\mathrm{d}_{1}=-0.1\right.$ and $\left.\delta=0.9\right)$ & 3.813 & 3.395 & 2.273 & 1.947 \\
\hline
\end{tabular}

For $d_{1}=0, h_{a}=1.0$, by choosing various $d_{2}$ in Table 2 , the MAUB on $h_{b}$ of the delay are listed in Table 2. Note that $\mathrm{m}=3$ implies the number of delay decomposition. From Table 2, it can be seen that

(i) the obtained MADBs using Theorem 3.1 and Corollary 3.4 are greater than the ones using Theorem 3.5 in [2]; 
(ii) Corollary 3.4 can provide lower MADBs than Theorem 3.1 since Corollary 3.4 is a special case of Theorem 3.1. Moreover, it is also seen that in the case $d_{2} \geqslant 1.0$, some terms on the time-derivative of the delay in this paper can work while those in the existing ones cannot help for deriving less conservative stability criteria, which is thus reduced to the unknown case, that is, the fast-varying delay case. This feature shows the tuning parameter $\delta$ plays a key effect on the reduced conservatism.

Table 2: MADB on $h_{b}$ for various $d_{2}\left(h_{a}=1.0\right.$ and $\left.d_{1}=0\right)$ for Example 4.1.

\begin{tabular}{llllllll}
\hline Method $\mathrm{d}_{2}$ & 0.1 & 0.3 & 0.5 & 0.7 & 1.0 & 1.1 & 1.2 \\
\hline An et al. [2] $(\mathrm{m}=3)$ & 3.286 & 2.318 & 1.771 & 1.701 & 1.684 & 1.684 & 1.684 \\
Corollary 3.4 $(\delta=0.8)$ & 3.597 & 2.604 & 1.996 & 1.714 & 1.686 & 1.695 & 1.682 \\
Theorem 3.1 $(\delta=0.8)$ & 3.622 & 2.794 & 2.267 & 1.973 & 1.899 & 1.901 & 1.893 \\
\hline
\end{tabular}

As an example, we assume that $\tau(t)=1.906+0.906 \sin (3 t / 9.06)$. Clearly, $1 \leqslant \tau(t) \leqslant 2.812$ and $-0.3 \leqslant \dot{\tau}(t) \leqslant 0.3$. Let $\delta=0.8$, by applying Theorem 3.1 in this paper, we can obtain the following solutions.

$$
\begin{array}{rlrl}
\mathrm{P}_{11} & =\left[\begin{array}{ll}
0.0182 & 0.0002 \\
0.0002 & 0.0015
\end{array}\right], & \mathrm{P}_{12}=10^{-3}\left[\begin{array}{cc}
0.2501 & -0.1280 \\
0.0834 & 0.2129
\end{array}\right], & \mathrm{P}_{13}=10^{-3}\left[\begin{array}{cc}
0.7602 & -0.0077 \\
-0.0077 & 0.0660
\end{array}\right], \\
\mathrm{P}_{21}=\left[\begin{array}{ll}
0.0184 & 0.0002 \\
0.0002 & 0.0016
\end{array}\right], & \mathrm{P}_{22}=10^{-3}\left[\begin{array}{cc}
0.0449 & -0.1747 \\
0.1180 & 0.2397
\end{array}\right], & \mathrm{P}_{23}=10^{-3}\left[\begin{array}{cc}
1.1747 & 0.0081 \\
0.0081 & 0.1305
\end{array}\right], \\
\mathrm{P}_{\delta}=10^{-3}\left[\begin{array}{ll}
1.8209 & 0.0072 \\
0.0072 & 0.2004
\end{array}\right], & \mathrm{Q}_{1}=\left[\begin{array}{ll}
0.0352 & 0.0011 \\
0.0011 & 0.0013
\end{array}\right], & \mathrm{Q}_{2}=\left[\begin{array}{cc}
0.0305 & 0.0013 \\
0.0013 & 0.0014
\end{array}\right], \\
\mathrm{Q}_{3}=\left[\begin{array}{ll}
0.0270 & 0.0017 \\
0.0017 & 0.0017
\end{array}\right], & \mathrm{Q}_{4}=10^{-3}\left[\begin{array}{cc}
3.8651 & -0.0546 \\
-0.0546 & 0.4371
\end{array}\right], & \mathrm{R}_{1}=10^{-3}\left[\begin{array}{cc}
1.1306 & -0.1176 \\
-0.1176 & 0.2227
\end{array}\right], \\
\mathrm{R}_{2}=10^{-3}\left[\begin{array}{cc}
1.3103 & 0.1539 \\
0.1539 & 0.2129
\end{array}\right], & \mathrm{R}_{3}=10^{-3}\left[\begin{array}{cc}
2.5117 & 0.1025 \\
0.1025 & 0.2662
\end{array}\right], & \mathrm{R}_{4}=10^{-3}\left[\begin{array}{cc}
1.5895 & 0.1280 \\
0.1280 & 0.2219
\end{array}\right], \\
\mathrm{S}_{1}=10^{-3}\left[\begin{array}{cc}
0.2179 & -0.0048 \\
-0.0048 & 0.0732
\end{array}\right], & \mathrm{S}_{2}=10^{-4}\left[\begin{array}{cc}
0.9657 & -0.0095 \\
-0.0095 & 0.5582
\end{array}\right], & \mathrm{S}_{3}=10^{-3}\left[\begin{array}{cc}
0.1882 & -0.0072 \\
-0.0072 & 0.0016
\end{array}\right], \\
\mathrm{S}_{4}=10^{-4}\left[\begin{array}{cc}
0.8900 & -0.0277 \\
-0.0277 & 0.0191
\end{array}\right], & \mathrm{R}_{\mathrm{s}}=10^{-4}\left[\begin{array}{cc}
0.0501 & 0.0885 \\
0.0885 & 1.6600
\end{array}\right] . & &
\end{array}
$$

Furthermore, with initial state condition $\phi(t)=[-0.5,0.8]^{\top}$, Fig. 1 depicts the state responses of the system, from which it is clear that the system is asymptotically stable.

Furthermore, we will give other examples to show the effectiveness and merit of nonlinear system via T-S fuzzy models, and the proposed approach also yields less conservative than the existing results.

Example 4.2 (Example 1 of [18]). Consider the following time-delayed nonlinear system:

$$
\begin{aligned}
& \dot{x}_{1}(t)=0.5\left(1-\sin ^{2}(\theta(t))\right) x_{2}(t)-x_{1}(t-\tau(t))-\left(1+\sin ^{2}(\theta(t))\right) x_{1}(t), \\
& \dot{x}_{2}(t)=\operatorname{sgn}\left(|\theta(t)|-\frac{\pi}{2}\right)\left(0.9 \cos ^{2}(\theta(t))-1\right) x_{1}(t-\tau(t))-x_{2}(t-\tau(t))-\left(0.9+0.1 \cos ^{2}(\theta(t))\right) x_{2}(t),
\end{aligned}
$$

which can be exactly expressed as a T-S delayed system (2.2) with the following rules [18, 20, 21, 25].

$$
\begin{aligned}
& R^{1}: \text { if } \theta(t) \text { is } \pm \frac{\pi}{2} \text {, then } \dot{x}(t)=A_{1} x(t)+A_{\tau 1} x(t-\tau(t)), \\
& R^{2}: \text { if } \theta(t) \text { is } 0 \text {, then } \dot{x}(t)=A_{2} x(t)+A_{\tau 2} x(t-\tau(t)) .
\end{aligned}
$$

The membership functions for rules 1 and 2 are $h_{1}\left(x_{1}(t)\right)=\sin ^{2}\left(x_{1}(t)\right), h_{2}\left(x_{1}(t)\right)=\cos ^{2}\left(x_{1}(t)\right)$ with the following parameters:

$$
A_{1}=\left[\begin{array}{cc}
-2 & 0 \\
0 & -0.9
\end{array}\right], \quad A_{\tau 1}=\left[\begin{array}{cc}
-1 & 0 \\
-1 & -1
\end{array}\right], \quad A_{2}=\left[\begin{array}{cc}
-1 & 0.5 \\
0 & -1
\end{array}\right], \quad A_{\tau 2}=\left[\begin{array}{cc}
-1 & 0 \\
0.1 & -1
\end{array}\right] .
$$




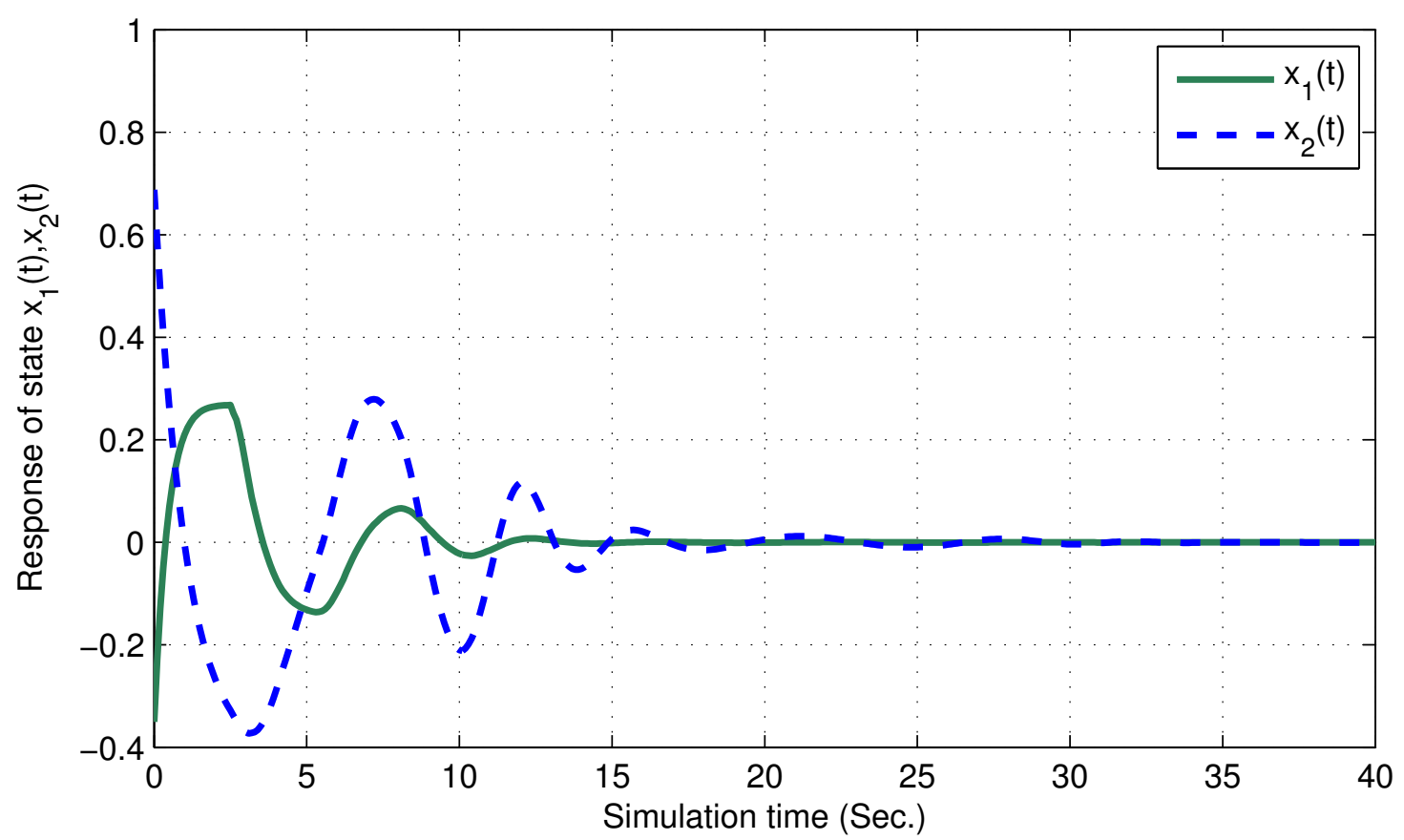

Figure 1: State responses of the system for Example 4.1.

For various $h_{a}$, the MADB on $h_{b}$ for guaranteeing the above system asymptotically stable are listed in Table 3. It is worth noting that in the case $d_{2} \geqslant 1.0$, in the existing ones the delay are often limited as the unknown case, that is, the fast-varying delay case, while the present results can work as before when $\mathrm{d}_{2} \geqslant 1$.0. From Table 3 , it can be seen that the proposed method yields less conservative that the exiting literatures $[2,12,18,20,21,25,27]$.

Table 3: MADB on $h_{b}$ for varying $h_{a}$ with $d_{2} \geqslant 1$ (or $d_{2}$ unknown) for Example 4.2.

\begin{tabular}{lllll}
\hline Method $\mathrm{h}_{\mathrm{a}}$ & 0.4 & 0.8 & 1.0 & 1.2 \\
\hline Lien et al. [12] & 0.8829 & 1.0677 & 1.1874 & 1.3181 \\
Peng et al. [21] & 1.0183 & 1.1817 & 1.2776 & 1.3816 \\
Peng and Han [20] & 1.18 & 1.31 & 1.37 & 1.43 \\
Tian et al. [27] & 1.2647 & 1.3032 & 1.3528 & 1.4214 \\
An et al. [2] & 1.277 & 1.311 & 1.358 & 1.419 \\
Peng and Fei [18] & 1.32 & 1.32 & 1.38 & 1.42 \\
Souza et al. [25] & 1.2836 & 1.3394 & 1.4009 & 1.4815 \\
Theorem 3.1 $\left(\delta=0.9, \mathrm{~d}_{1}=0, \mathrm{~d}_{2}=1.1\right)$ & 1.6998 & 1.6561 & 1.6469 & 1.6413 \\
\hline
\end{tabular}

Furthermore, to compare with the existing results in $[10,12,13,21,32]$, we assume that $h_{a}=0$. Using Theorem 3.5 yields MADB on $h_{b}$ greater than those in [10, 12, 13, 21, 32], which are listed in Table 4. It is worth mentioning that the tuning parameter can play a key effect on reduced conservatism of the proposed results, and the existing method in [10,12, 13, 21, 32] can only mean the delay is not differential, that is, the time-derivative of the delay is unknown, while the proposed method can still show that $d_{2} \geqslant 1.0$ is effective for reducing the conservatism. 
Table 4: MADB on $h_{b}$ for varying $d_{2}$ with $h_{a}=0$ for Example 4.2.

\begin{tabular}{llll}
\hline Method $\mathrm{d}_{2}$ & 0.0 & 0.1 & $\geqslant 1.0$ \\
\hline Lien et al. [12] & 1.5973 & 1.4840 & 0.8310 \\
Liu et al. [13] & 1.5974 & 1.4957 & 1.2642 \\
Peng et al. [21] & 1.8034 & & 0.9899 \\
Kwon et al. [10] & 1.6609 & 1.5332 & 1.2696 \\
Zeng et al. [32] & 2.0002 & 1.8090 & 1.3631 \\
Theorem 3.5 $\left(\mathrm{d}_{1}=-0.1\right)$ & $2.0214(\delta=0.5)$ & $1.8943(\delta=0.6)$ & $1.4239\left(\delta=0.7, \mathrm{~d}_{2}=1.1\right)$ \\
\hline
\end{tabular}

\section{Conclusion}

The problem of stability analysis for interval delayed T-S fuzzy systems is investigated. By choosing some delay-dependent LK functional, new delay-derivative-dependent and delay-fractional-dependent criteria are derived in terms of LMIs. The obtained conditions produce better results than some existing ones, since the variable delay decomposition technique and new integral inequalities are included. Finally, the superiority of the present result has been validated through numerical examples.

\section{Acknowledgment}

The work described in this paper was supported in part by the National Natural Science Foundation of China (Grant No.61370097), and in part by the National Science Fund for Distinguished Young Scholars in China (Grant No.11225212).

\section{References}

[1] J.-Y. An, T. Li, G.-L. Wen, R.-F. Li, New stability conditions for uncertain TS fuzzy systems with interval time-varying delay, Int. J. Control Autom. Syst., 10 (2012), 490-497. 1, 4, 4.1, 1

[2] J.-Y. An, G.-L. Wen, Improved stability criteria for time-varying delayed T-S fuzzy systems via delay partitioning approach, Fuzzy Sets and Systems, 185 (2011), 83-94. 1, 3.3, 3.7, 4, 4.1, 2, 4.2, 3

[3] J.-Y. An, G.-L. Wen, C. Lin, R.-F. Li, New results on delay-derivative-dependent fuzzy $H^{\infty}$ filter design for T-S fuzzy systems, IEEE Trans. Fuzzy Syst., 19 (2011), 770-779. 1

[4] J.-Y. An, G.-L. Wen, W. Xu, Improved results on fuzzy $\mathrm{H} \infty$ filter design for T-S fuzzy systems, Discrete Dyn. Nat. Soc., 2010 (2010), 21 pages. 1

[5] C. Briat, Convergence and equivalence results for the Jensen's inequality-application to time-delay and sampled-data systems, IEEE Trans. Automat. Control, 56 (2011), 1660-1665. 1

[6] Y.-Y. Cao, P. M. Frank, Stability analysis and synthesis of nonlinear time-delay systems via linear Takagi-Sugeno fuzzy models, Fuzzy Sets and Systems, 124 (2001), 213-229. 1

[7] F. A. Faria, G. N. Silva, V. A. Oliverira, Reducing the conservatism of LMI-based stabilisation conditions for TS fuzzy systems using fuzzy Lyapunov functions, Internat. J. Systems Sci., 44 (2013), 1956-1969. 1, 3.2

[8] K. Gu, V. L. Kharitonov, J. Chen, Stability of time-delay systems, Control Engineering, Birkhäuser Boston, Inc., Boston, MA, (2003). 1, 3

[9] J. K. Hale, S. M. Verduyn Lunel, Introduction to functional-differential equations, Applied Mathematical Sciences, Springer-Verlag, New York, (1993). 1, 3, 3

[10] O. M. Kwon, M. J. Park, S. M. Lee, J. H. Park, Augmented Lyapunov-Krasovskii functional approaches to robust stability criteria for uncertain Takagi-Sugeno fuzzy systems with time-varying delays, Fuzzy Sets and Systems, 201 (2012), 1-19. $3.2,3.3,4,4.2,4$

[11] D. H. Lee, Relaxed LMI conditions for local stability and local stabilization of continuous-time TakagiSugeno fuzzy systems, IEEE Trans. Cybern., 44 (2014), 394-405. 1, 3.2

[12] C.-H. Lien, K.-W. Yu, W.-D. Chen, Z.-L. Wan, Y.-J. Chung, Stability criteria for uncertain Takagi-Sugeno fuzzy systems with interval time-varying delay, IET Control Theory Appl., 1 (2007), 764-769. 1, 3.3, 4, 4.2, 3, 4.2, 4

[13] F. Liu, M. Liu, Y. He, R. Yokoyama, New delay-dependent stability criteria for T-S fuzzy systems with time-varying delay, Fuzzy Sets and Systems., 161 (2010), 2033-2042. 1, 3.3, 4, 4.1, 1, 4.2, 4

[14] Y. S. Moon, P. G. Park, W. H. Kwon, Y. S Lee, Delay-dependent robust stabilization of uncertain state-delayed systems, Internat. J. Control, 74 (2001), 1447-1455. 1, 2.1 
[15] M. Narimani, H.-K. Lam, R. Dilmaghani, C. Wolfe, LMI-based stability analysis of fuzzy-model-based control systems using approximated polynomial membership functions, IEEE Trans. Syst., Man, Cybern. B, 41 (2011), 713-724. 1

[16] P. G. Park, J. W. Ko, C.-K. Jeong, Reciprocally convex approach to stability of systems with time-varying delays, Automatica J. IFAC, 47 (2011), 235-238. 1, 3

[17] P. G. Park, W. I. Lee, S. Y. Lee, Auxiliary function-based integral inequalities for quadratic functions and their applications to time-delay systems, J. Franklin Inst., 352 (2015), 1378-1396. 1, 1, 2.3

[18] C. Peng, M.-R. Fei, An improved result on the stability of uncertain T-S fuzzy systems with interval time-varying delay, Fuzzy Sets and Systems, 212 (2013), 97-109. 1, 3.3, 4.2, 3

[19] C. Peng, M.-R. Fei, E.-G. Tian, Networked control for a class of T-S fuzzy systems with stochastic sensor faults, Fuzzy Sets and Systems, 212 (2013), 62-77. 1

[20] C. Peng, Q.-L. Han, Delay-range-dependent robust stabilization for uncertain T-S fuzzy control systems with interval time-varying delays, Inform. Sci., 181 (2011), 4287-4299. 1, 3.3, 4, 4.2, 3

[21] C. Peng, L.-Y. Wen, J.-Q. Yang, On delay-dependent robust stability criteria for uncertain T-S fuzzy systems with interval time-varying delay, Int. J. Fuzzy Syst., 13 (2011), 35-44. 1, 4, 4.2, 3, 4.2, 4

[22] J.-B. Qiu, G. Feng, H.-J. Gao, Fuzzy-model-based piecewise $\mathrm{H}_{\infty}$ static-output-feedback controller design for networked nonlinear systems, IEEE Trans. Fuzzy Syst., 18 (2010), 919-934. 1, 3.3

[23] A. Seuret, F. Gouaisbaut, Wirtinger-based integral inequality: application to time-delay systems, Automatica J. IFAC, 49 (2013), 2860-2866. 1, 3.2

[24] A. Seuret, F. Gouaisbaut, Complete quadratic Lyapunov functionals using Bessel-Legendre inequality, Proceedings of European Control Conference, (2014), 448-453. 1

[25] F. O. Souza, V. C. S. Campos, R. M. Palhares, On delay-dependent stability conditions for Takagi-Sugeno fuzzy systems, J. Franklin Inst., 351 (2014), 3707-3718. 1, 3.3, 4, 4.2, 3

[26] T. Takagi, M. Sugeno, Fuzzy identification of systems and its applications to modeling and control, IEEE Trans. Syst., Man, Cybern. 1 (1985), 116-132. 1

[27] E.-G. Tian, D. Yue, Y.-J. Zhang, Delay-dependent robust $\mathrm{H}_{\infty}$ control for T-S fuzzy system with interval time-varying delay, Fuzzy Sets and Systems, 160 (2009), 1708-1719. 1, 3.3, 4, 4.2, 3

[28] L.-G. Wu, X.-J. Su, P. Shi, J.-B. Qiu, A new approach to stability analysis and stabilization of discrete-time TS fuzzy time-varying delay systems, IEEE Trans. Syst., Man, Cybern. B, 41 (2011), 273-286. 1

[29] X.-P. Xie, S.-L. Hu, Relaxed stability criteria for discrete-time TakagiSugeno fuzzy systems via new augmented nonquadratic Lyapunov functions, Neurocomputing, 166 (2015), 416-421. 1

[30] X.-P. Xie, S.-X. Weng, H.-F. Zhang, Reducing the conservatism of stability analysis for discrete-time TS fuzzy systems based on a delayed Lyapunov function, Neurocomputing, 171 (2016), 1139-1145. 3.3

[31] J. Yang, W.-P. Luo, K.-B. Shi, X. Zhao, Robust stability analysis of uncertain T-S fuzzy systems with time-varying delay by improved delay-partitioning approach, J. Nonlinear Sci. Appl., 9 (2016), 171-185. 1

[32] H.-B. Zeng, J. H. Park, J.-W. Xia, S.-P. Xiao, Improved delay-dependent stability criteria for T-S fuzzy systems with time-varying delay, Appl. Math. Comput., 235 (2014), 492-501. 1, 3.2, 3.3, 3.7, 4, 4.1, 1, 4.2, 4

[33] X.-M. Zhang, Q.-L. Han, Novel delay-derivative-dependent stability criteria using new bounding techniques, Internat. J. Robust Nonlinear Control, 23 (2013), 1419-1432. 1, 3.6, 3.7, 4

[34] Z.-Y. Zhang, C. Lin, B. Chen, New stability and stabilization conditions for T-S fuzzy systems with time delay, Fuzzy Sets and Systems, 263 (2015), 82-91. 1, 3.3 